\title{
The Magnetic Field of the Irregular Galaxy NGC 4214
}

\author{
Amanda A. Kepley ${ }^{1}$, Ellen G. Zweibel, Eric M. Wilcots \\ Washburn Observatory, University of Wisconsin, 475 North Charter Street, Madison, WI \\ 53706 \\ kepley@astro.wisc .edu, zweibel@astro.wisc .edu, ewilcots@astro.wisc .edu \\ Kelsey E. Johnson \\ Department of Astronomy, University of Virginia, P.O. Box 400325, Charlottesville, VA \\ 22904, USA ; Adjunct at National Radio Astronomy Observatory, 520 Edgemont Road, \\ Charlottesville, VA 22903, USA. \\ kej7a@virginia.edu \\ Timothy Robishaw \\ Sydney Institute for Astronomy, School of Physics, The University of Sydney, NSW 2006, \\ Australia \\ tim.robishaw@sydney.edu.au
}

\begin{abstract}
We examine the magnetic field in NGC 4214, a nearby irregular galaxy, using multi-wavelength radio continuum polarization data from the Very Large Array. We find that the global radio continuum spectrum shows signs that free-free absorption and/or synchrotron losses may be important. The $3 \mathrm{~cm}$ radio continuum morphology is similar to that of the $\mathrm{H} \alpha$ while the $20 \mathrm{~cm}$ emission is more diffuse. We estimate that $50 \%$ of the radio continuum emission in the center of the galaxy is thermal. Our estimate of the magnetic field strength is $30 \pm 9.5 \mu \mathrm{G}$ in the center and $10 \pm 3 \mu \mathrm{G}$ at the edges. We find that the hot gas, magnetic, and the gravitational pressures are all the same order of magnitude. Inside the central star forming regions, we find that the thermal and turbulent pressures
\end{abstract}

\footnotetext{
${ }^{1}$ Now at Department of Astronomy, University of Virginia, P.O. Box 400325, Charlottesville, VA 22904, USA; Visiting Research Associate at National Radio Astronomy Observatory, 520 Edgemont Road, Charlottesville, VA 22903, USA.
} 
of the HII regions dominate the pressure balance. We do not detect any significant polarization on size scales greater than 200 pc. We place an upper limit of $8 \mu \mathrm{G}$ on the uniform field strength in this galaxy. We suggest that the diffuse synchrotron region, seen to the north of the main body of emission at $20 \mathrm{~cm}$, is elongated due to a uniform magnetic field with a maximum field strength of $7.6 \mu \mathrm{G}$. We find that, while the shear in NGC 4214 is comparable to that of the Milky Way, the supernova rate is half that of the Milky Way and suggest that the star formation episode in NGC 4214 needs additional time to build up enough turbulence to drive an $\alpha-\omega$ dynamo.

Subject headings: galaxies: ISM - galaxies: individual (NGC 4214) - galaxies: irregular - galaxies: magnetic fields - polarization

\section{Introduction}

Modern theories of galaxy formation postulate a bottom-up process where smaller systems merge together to form successively larger structures like the spiral galaxies we see today (Springel et al. 2005). In the local universe, low-mass galaxies are analogs to these high-redshift "building blocks." By studying nearby low mass galaxies, in particular low mass irregular galaxies, we can gain detailed insight into the basic physical processes at work in these systems, which are different than those in spirals or ellipticals due to their smaller mass. Studying the interstellar medium (ISM) of irregular galaxies is particularly important because their ISM is strongly influenced by star formation and interactions, which significantly affect the future evolution of these galaxies.

There have been a number of surveys of low mass irregular galaxies aimed at investigating the properties of the neutral and ionized gas components of the ISM and the contributions to the ISM by the stellar components (e.g., Hunter \& Elmegreen 2004; Hunter et al. 2006; Hunter \& Elmegreen 2006; Hunter et al. 2007, 2010). Relatively little attention, however, has been paid to the magnetic field component of the interstellar medium.

In general, the magnetic field energy is thought to be in equipartition with the turbulent and cosmic ray energies (Zweibel \& Heiles 1997; Ferrière 2001; Cox 2005; although see Chi \& Wolfendale 1993 for a contrasting view) and is thus an important part of the interstellar medium. In the few irregular galaxies with measured magnetic fields (Havnes et al. 1986, 1991; Chvży et al. 2000; Chvzy et al. 2000; Chvży et al. 2003; Gaensler et al. 2005; Mao et al. 2008; Kepley et al. 2010; Chyży et al. 2011), the magnetic fields are comparable in strength to those in larger spirals like the Milky Way (Ferrière 2001; Wielebinski 2005) 
and M51 (Beck et al. 1987). Therefore, in irregular galaxies, the magnetic field may play a more important role in the dynamics of the ISM than in spiral galaxies. Magnetic fields also channel gas flows and distribute and accelerate cosmic rays (Beck 2005; Everett et al. 2008, 2010). Irregular galaxies have shallow potential wells that are easily disrupted and potentially have a significant amount of their mass expelled from the galaxy (Ferrara \& Tolstoy 2000; Tremonti et al. 2004). Magnetic fields may either help the baryons escape or help to confine them to the galaxy depending on the structure of the magnetic fields (Tomisaka 1990; Mineshige et al. 1993; de Avillez \& Breitschwerdt 2005; Everett et al. 2008, 2010). Finally, the presence of significant magnetic fields in irregular galaxies raises the question of how they were generated in the first place. The main ingredients in generating and sustaining galactic magnetic fields are generally thought to be large-scale shear and small-scale turbulence. The well known $\alpha-\omega$ dynamo models are based on these effects (although see Zweibel \& Heiles 1997, Kulsrud 1999, , and Kulsrud \& Zweibel 2008 for critiques of this model). In the $\alpha-\omega$ dynamo, differential rotation $(\omega)$ is used to stretch small-scale turbulence $(\alpha)$ leading to an increase in magnetic field strength and the presence of a large-scale magnetic field. It may be harder for $\alpha-\omega$ dynamos to operate in irregular galaxies because they rotate mostly as solid bodies and their ISM is more easily disrupted because of their smaller potential wells. The strength and structure of the magnetic fields in irregular galaxies provide stringent constraints for dynamo models of these objects.

At present, only a handful of nearby galaxies have detailed magnetic field measurements: NGC 1569 (Kepley et al. 2010), the Large and Small Magellanic Clouds (Haynes et al. 1986, 1991; Klein et al. 1993; Gaensler et al. 2005; Mao et al. 2008), NGC 4449 (Chyży et al. 2000), IC 10 (Chyży et al. 2003), NGC 6822 (Chyży et al. 2003), and IC 1613 (Chyży et al. 2011). To better understand the role of magnetic fields in the interstellar medium of irregular galaxies and how these fields are generated in these systems, we have embarked on a program to obtain deep, high-resolution radio continuum data on several irregular galaxies. In this first paper in this series (Kepley et al. 2010), we presented our observations of the magnetic field of NGC 1569, a dwarf irregular galaxy hosting one of the most extreme starbursts in the nearby universe. The magnetic field in this galaxy is shaped by its outflow and the field may be playing an important role in channeling gas away from the disk.

Work by other groups have found that the LMC, SMC, and NGC 4449 have large-scale ordered fields like NGC 1569. The LMC has a $1 \mu \mathrm{G}$ regular, axisymmetric spiral field, a random field of $4.1 \mu \mathrm{G}$, and a total field of $4.3 \mu \mathrm{G}$ (Gaensler et al. 2005). A cosmic raydriven dynamo (Parker 1992; Hanasz et al. 2004) has been invoked to explain the presence of a regular field despite the vigorous recent star formation in the LMC (Gaensler et al. 2005). The SMC has a random magnetic field strength of $3 \mu \mathrm{G}$ and a unidirectional, regular field with a strength of $1.7 \mu \mathrm{G}$ aligned roughly along the Magellanic bridge, which suggests 
an origin associated with the interaction between the LMC and SMC (Mao et al. 2008). In contrast to the weak, but regular fields in the LMC and SMC, NGC 4449 has a strong uniform field of $8 \mu \mathrm{G}$ and a total field of $14 \mu \mathrm{G}$ (Chyży et al. 2000). The uniform field shows a fan-like structure in the inner regions of the galaxy and a spiral structure in the outer regions. A fast dynamo model including velocity shear around a bar, a wind from the central region of the galaxy, and a random field produces a magnetic field configuration very similar to that of NGC 4449 (Otmianowska-Mazur et al. 2000). In contrast to the magnetic fields of NGC 1569, the LMC, the SMC, and NCG 4449, the uniform fields of IC 10, NGC 6822 , and IC 1613 (as traced by the polarization of their radio continuum emission) are either weak or non-existent (Chyży et al. 2003). IC 10 has a diffuse, random field with a strength of $14 \mu \mathrm{G}$ in the center and $7 \mu \mathrm{G}$ on its outskirts. NGC 6822 has a random field with a maximum strength of $5 \mu \mathrm{G}$ and IC 1613 has a random magnetic field of $2.8 \mu \mathrm{G}$. Both IC 10 and NGC 6822 have small patches of uniform fields with strengths of $2-3 \mu \mathrm{G}$ on size scales much smaller than the size of the galaxy. Chyży et al. (2003) suggest that the fields in these galaxies are the result of a fluctuating dynamo (Subramanian 1998). IC 1613 shows no sign of uniform field; any polarized emission can be attributed to background sources (Chyży et al. 2011).

The line of sight fields determined by Gaensler et al. (2005) and Mao et al. (2008) for the LMC and SMC are based on the rotation measures of background sources, while the results for NGC 4449, IC 10, NGC 6822, IC 1613, and NGC 1569 are based on observations of the radio continuum polarization. There are significant differences between the two methods. The radio continuum polarization observations trace the magnetic field with cosmic ray electrons, while the rotation measure observations trace the field with thermal electrons. In addition, only the rotation measure observations can tell a regular (i.e., coherent) field (all one direction) from a uniform field (one orientation, but the direction uncertain by $180^{\circ}$ ). Unfortunately, current technology limits this method to nearby galaxies (e.g., LMC, SMC, M31) with very large angular sizes, although this situation will improve with the development of the Square Kilometer Array (Beck \& Gaensler 2004).

In this paper, we focus on NGC 4214, which is classified as a mixed, S-shaped, Magellanic irregular galaxy by de Vaucouleurs et al. (1995). NGC 4214 is a well-studied galaxy whose rest-frame UV spectrum strongly resembles that of star-forming galaxies at $z=3$ (Steidel et al. 1996). Observations of its stars and ionized gas (Maiz-Apellaniz et al. 1998; MacKenty et al. 2000; Drozdovsky et al. 2002; Úbeda et al. 2007a., b) show that there are two active regions of star formation in the galaxy: NGC 4214-I (also referred to as the northwest complex) and NGC 4214-II (also referred to as the southeast complex). NGC 4214-I is the older complex with an age of 3 to $4 \mathrm{Myr}$ and consists of an $\mathrm{H} \alpha$ shell surrounding a super-star cluster (MacKenty et al. 2000). The H $\alpha$ kinematics of NGC 4214-I show some indications of 
expanding shells (Martin 1998; Maíz-Apellániz et al. 1999; Wilcots \& Thurow 2001). NGC 4214-II is slightly younger with an age of 2.5 to 3.0 Myr (MacKenty et al. 2000). The H $\alpha$ in this region is coincident with the star cluster, showing that the cluster has not had sufficient time to clear out a large H $\alpha$ cavity (MacKenty et al. 2000). Schwartz \& Martin (2004) detect an outflow of cold gas in this region with a velocity of $23 \mathrm{~km} \mathrm{~s}^{-1}$, which is associated with a small $\mathrm{H} \alpha$ bubble. X-ray observations with Chandra and XMM-Newton show that the hot gas in this galaxy is centered on the star forming regions (Hartwell et al. 2004; Ott et al. 2005a,b). The star forming regions of NGC 4214 and their associated ionized and hot gas are embedded in a disk of much older stars (Fanelli et al. 1997; Drozdovsky et al. 2002), which in turn is embedded in a neutral hydrogen envelope which extends to 1.4 times the Holmberg radius (5.3') 1 of this galaxy (Allsopp 1979; McIntyre 1997).

The distance to NGC 4214 is controversial, with distances used in the literature ranging from $2 \mathrm{Mpc}$ (Hopp et al. 1999) to $7 \mathrm{Mpc}$ (Allsopp 1979). We adopt a distance of $2.94 \mathrm{Mpc}$ in this paper, which is the distance for the galaxy determined from Hubble Space Telescope imaging of the tip of the red giant branch by Maíz-Apellániz et al. (2002). This distance agrees with the tip of the red giant branch distances determined independently by Drozdovsky et al. (2002, 2.70 Mpc) and Dalcanton et al. (2009, 3.04 Mpc) and with the distance determined using the planetary nebula luminosity function by Dopita et al. (2010, $3.19 \mathrm{Mpc}$ ). It is also in reasonable agreement with distances determined by its radial velocity and models of the Hubble flow determined by NED2 2.75 to $7.53 \mathrm{Mpc})$. At the adopted distance, $1^{\prime \prime}$ is $14.2 \mathrm{pc}$.

The observations presented in this paper represent the most sensitive observations of the radio continuum emission of NGC 4214 to date. Previous observations of the radio continuum of NGC 4214 include Allsopp (1979), Beck et al. (2000), and MacKenty et al. (2000). Although the resolution of our observations is lower than that of Beck et al. (2000) and MacKenty et al. (2000), we integrated for substantially longer (effective integration times ranging from 5 hours to 15 hours rather than 15 minutes). Our choice of array configuration (see Section 21) allows us good sensitivity to both small scale features and large-scale diffuse emission in NGC 4214. Our observations trace the thermal emission produced by the ionizing radiation of young stars and the synchrotron emission produced by the detonation of old massive stars, which nicely complements the new data on the stellar content of NGC 4214 obtained by the Wide Field Camera 3 on the Hubble Space Telescope (e.g., Dopita et al.

\footnotetext{
${ }^{1}$ The Holmberg radius is the radius where the surface brightness of a galaxy falls to 26.5 mag $\operatorname{arcsec}^{-2}$.

${ }^{2}$ The NASA/IPAC Extragalactic Database (NED) is operated by the Jet Propulsion Laboratory, California Institute of Technology, under contract with the National Aeronautics and Space Administration.
} 
2010) and the new data on the molecular components of the ISM obtained using Herschel (e.g., Cormier et al. 2010).

We detail our data calibration and imaging process in Section 2. In Section 3, we present our observations in detail. Section 4 is devoted to our results including a comparison with multi-wavelength data (Section 4.1), estimates of the thermal fraction of the radio continuum emission (Section 4.2), an estimation of the magnetic field strength in NGC 4214 (Section 4.3), an analysis of the importance of the magnetic field in the ISM of NGC 4214 (Section 4.4), an investigation of the magnetic field structure of NGC 4214 (Section 4.5), estimates of cosmic ray lifetimes in NGC 4214 (Section 4.6), and a discussion of possible mechanisms for generating the field (Section 4.7). Finally, we summarize our conclusions in Section 5 ,

\section{Data}

We have obtained radio continuum polarization observations of NGC 4214 at 20, 6, and $3 \mathrm{~cm}$ using the National Radio Astronomy Observatory (NRAO) Very Large Array (VLA) 3 Our observations (proposal IDs: AK606, AK616; PI: Kepley) were taken between 2005 August and 2005 December. See Table 1 for a summary of these observations. We used two different configurations of the VLA to as closely as possibly match the synthesized beams at our three different observing frequencies. The correlator was configured for two intermediate frequencies (IFs) each with $50 \mathrm{MHz}$ bandwidth and full polarization products (RR, LL, RL, LR). For all our observations, we used 1331+305 (3C286) to calibrate the flux density scale and absolute polarization angle and the relevant secondary calibrator to calibrate the amplitudes and phases as well as the instrumental polarization.

For our $20 \mathrm{~cm}$ observations, we observed our secondary calibrator $(1227+365)$ for four minutes for every thirty-five minutes on source. The primary calibrator $(1331+305)$ was observed for ten minutes near the beginning of the observations and ten minutes at the end of the observations. For our $6 \mathrm{~cm}$ observations, we observed each pointing of a four pointing mosaic for eight minutes and observed the secondary calibrator for four minutes once every cycle through the mosaic. The primary calibrator was observed in a fashion similar to that of our $20 \mathrm{~cm}$ observations. For our $3 \mathrm{~cm}$ observations, we observed each pointing of a sixteen pointing mosaic for six minutes. Every half cycle through the mosaic we observed our secondary calibrator $(1146+399)$ for six minutes. The primary calibrator observations

\footnotetext{
${ }^{3}$ The National Radio Astronomy Observatory is a facility of the National Science Foundation operated under cooperative agreement by Associated Universities, Inc.
} 
were the same as in our $20 \mathrm{~cm}$ and $6 \mathrm{~cm}$ observations.

We calibrated the data in AIPS. Our data reduction procedures followed the standard procedures to calibrate VLA data outlined in the AIPS Cookbook (Greisen 2008) with some additional effort to properly calibrate the polarization. We briefly sketch our data reduction procedures below.

After the initial flagging of the data, we used the primary calibrator (with the appropriate $u$ - $v$ limits) to set the primary flux density scale. With our observational setup, 3C286 $(1331+305)$ is well-approximated as a point source. We then calibrated the amplitudes and phases of the primary and secondary calibrators. The flux density value for the secondary calibrator was bootstrapped from the flux density value obtained for the primary calibrator. We applied the calibrated amplitudes and phases to their respective calibrators and the calibrated amplitudes and phases from the secondary calibrator to the source. To calibrate the instrumental polarization response, we used our observations of the secondary calibrator. The secondary calibrator was observed at least five times over at least 100 degrees of parallactic angle, which allows us to use the rotation of the feed with respect to the sky to separate the instrumental polarization (which does not rotate as the parallactic angle changes) from the astronomical polarization (which does rotate as the parallactic angle changes). The calibration for the instrumental polarization was then applied to the data. The primary calibrator was used to determine the absolute polarization angle and this final correction applied to the data.

After calibrating in AIPS, we exported the I, Q, and U data as FITS files and imaged the data in Miriad (Sault et al. 1995) to take advantage its ability to jointly deconvolve mosaics. For the $3 \mathrm{~cm}$ and $6 \mathrm{~cm}$ data, we used the mosaic option on the invert task to combine the different pointings of the mosiac. The data was weighted with a robust value of 0 . For the $3 \mathrm{~cm}$ data, we tapered the data in the $u-v$ plane to match the resolution of the data at $20 \mathrm{~cm}$ and $6 \mathrm{~cm}$. We then cleaned the mosaic using the task mossdi. Since the $20 \mathrm{~cm}$ data was only a single pointing, we used invert without the mosaic option to image the data and the task clean to clean the resulting image. In the $20 \mathrm{~cm}$ data, there was a bright source to the southwest of the galaxy that we were not able to completely model during cleaning. The incomplete removal of the source has the side-effect of increasing the noise in the $20 \mathrm{~cm}$ Stokes I image to approximately 4 times the theoretical noise at $20 \mathrm{~cm}$ and twice the noise in the other images. The Stokes $\mathrm{Q}$ and $\mathrm{U}$ images were not affected as severely and their noise levels are much closer to the theoretical noise. Table 2 summarizes the properties of our final images. 


\section{Properties of the Radio Continuum Emission}

\subsection{Total Intensity}

Figure 1 shows the distribution of the radio continuum emission in NGC 4214 . The emission is most extended at $20 \mathrm{~cm}$ despite the higher noise levels in the $20 \mathrm{~cm}$ image. At $3 \mathrm{~cm}$, the emission is largely confined to two regions near the center of the galaxy. The circles in the middle and right panels of Figure 1 show the largest angular scale imaged by the VLA at $6 \mathrm{~cm}$ and $3 \mathrm{~cm}$. The largest angular scale imaged by the VLA at $20 \mathrm{~cm}$ is larger than the region shown in Figure 1 .

We recover most of the radio continuum flux from the galaxy. The first evidence in favor of this conclusion is that the extent of NGC 4214 at both $6 \mathrm{~cm}$ and $3 \mathrm{~cm}$ is much smaller than the largest angular scale imaged at those wavelengths. The second line of evidence for this conclusion is shown in Figure 2. In this Figure, we plot the sum of the $3 \mathrm{~cm}$ radio continuum emission in a particular annulus as a function of radius from the center of the galaxy. The presence of a large negative bowl surrounding the radio continuum emission would indicate that large-scale emission is being resolved out. This effect would be largest for the $3 \mathrm{~cm}$ data because it will resolve out emission on smaller scales than the $6 \mathrm{~cm}$ or $20 \mathrm{~cm}$ data. There is no significant negative bowl surrounding our $3 \mathrm{~cm}$ radio continuum emission. The dip at $150^{\prime \prime}$ is well beyond the main body of radio continuum emission and is the same magnitude as the variations at larger radii. Finally, we have plotted the total flux from our data along with measurements of the total flux from the literature in Figure 3. We assume that the errors on our total fluxes and those of measurements from the literature without quoted errors are $20 \%$. This error reflects the fundamental uncertainty in the calibration of radio fluxes. Table 3 lists the values and their sources. Our measured radio continuum fluxes are larger than the previously measured radio continuum fluxes. Therefore, we are not resolving out significant emission on scales larger than our largest angular scale. Our measured fluxes are larger than the fluxes from previous observations because all of the previous observations were shallow survey observations and these observations are able to detect more faint diffuse emission.

Given the systematic offset between the archival measurements and our data, the heterogeneity of the archival measurements, and the limited number of measurements from our observations, we only fit a simple model consisting of a single power law to our radio continuum spectrum for NGC 4214. We find that the data can be fit by a single power law with a spectral index 4 equal to $-0.43 \pm 0.06$. This spectral index shows that the spectrum includes

\footnotetext{
${ }^{4}$ We define the spectral index, $\alpha$, as $S_{\nu} \propto \nu^{\alpha}$.
} 
a mix of thermal and non-thermal emission. We see some hint that the spectrum is convex, which would suggest that models including free-free absorption of synchrotron and/or freefree emission or including synchrotron losses might be appropriate (Deeg et al. 1993). The electron densities measured in NGC 4214 by Kobulnicky \& Skillman (1996) are similar to the electron densities determined by the modeling of the radio continuum spectra of other similar galaxies including free-free absorption of synchrotron emission by Deeg et al. (1993). However, our limited data set does not justify the use of more complex models. Additional data points at higher and lower frequencies determined using data that matches the spatial frequency range and sensitivity of our data are necessary to model these more complex physical scenarios.

There are two isolated regions of radio continuum emission located to the north and northeast of the main emission region. The first is a region of diffuse $20 \mathrm{~cm}$ emission to the north of the galaxy at $\left(12^{\mathrm{h}} 15^{\mathrm{m}} 38^{\mathrm{s}}, 36^{\circ} 22^{\prime} 30^{\prime \prime}\right)$, referred to hereafter as the diffuse synchrotron region. This region is likely to be part of NGC 4214 based on the presence of the optical and $\mathrm{H} \alpha$ emission in the same location (see $\$ 4.1$ for further discussion). The bright radio continuum source with a steep radio continuum spectrum (see $\S[3.2)$ at $\left(12^{\mathrm{h}} 15^{\mathrm{m}} 48.9^{\mathrm{s}}, 36^{\circ} 21^{\prime} 54.10^{\prime \prime}\right)$ is resolved into two sources by the Faint Images of the Radio Sky at Twenty centimeters (FIRST) survey (Becker et al. 1995). McMahon et al. (2002) is able to identify an optical counterpart for this source on the red POSS-I plates; the optical morphology is classified as blended (two local maxima with a single set of connected above-threshold pixels). Unfortunately, there are no determinations of the source redshift. The southernmost of the two FIRST sources is coincident with x-ray point source 20 from Hartwell et al. (2004). The x-ray emission from this source is extremely hard $((H-M) /(H+M)=0.698$, $(M-S) /(M+S)=0.231)$. Based on the steep spectra index in the radio, the blended source identification of McMahon et al. (2002), and the hard x-ray colors from the associated x-ray source, we conclude that this source is a background AGN.

\subsection{Spectral Indices}

The spectral indices between the $20 \mathrm{~cm}$ and the $6 \mathrm{~cm}$ data and the $6 \mathrm{~cm}$ and the $3 \mathrm{~cm}$ data are shown in Figure 4. Regions in the input images below $3 \sigma$ were masked prior to calculating the spectral index. Thermal emission has a spectral index of -0.1 and synchrotron

emission has a spectral index of $\sim-0.7$, although free-free absorption and synchrotron losses can modify the observed spectrum. The two brightest emission peaks are thermally dominated with the younger (2.5 to 3.0 Myr; MacKenty et al. 2000), southern peak (NGC 4214-II) having a flatter spectral index than the older (3.0 to 4.0 Myr; MacKenty et al. 
2000), northern peak (NGC 4214-I). Flat radio continuum spectra like those seen in NGC 4214-I and NGC 4214-II are characteristic of dwarf starburst galaxies (Klein et al. 1984; Deeg et al. 1993). The two fainter sources northwest of the galaxy (at $12^{\mathrm{h}} 15^{\mathrm{m}} 34^{\mathrm{s}}, 36^{\circ} 20^{\prime}$ ) are also thermally dominated, but with a less steep spectrum than NGC 4214-I and NGC 4214-II. The regions between NGC 4214-I and -II and the peaks to the northwest have steeper spectra showing that synchrotron emission plays a more important role in these regions.

\subsection{Polarized Emission}

Since we were interested in investigating the structure of the magnetic field, we determined the polarized intensity and the polarization angle from the Stokes Q and U images. The derived polarized intensities were corrected for the bias in the estimated polarized intensity (Simmons \& Stewart 1985; Vaillancourt 2006).

The percent polarization gives the strength of the uniform field relative to that of the total field, while the polarization angle rotated by $\sim 90^{\circ}$ gives the orientation of the magnetic field. The polarized emission of NGC 4214 is shown in Figure 5. There is little polarized emission associated with NGC 4214. This lack of polarization could be due to several instrumental/imaging effects or due to depolarization effects internal to the galaxy. The possible instrumental imaging effects are: 1) depolarization due to mosaicing, 2) the polarized emission is stronger at lower resolutions, or 3) bandwidth depolarization due to combining two IFs. To check that mosaicing the observations has not depolarized the final image or produced spurious polarization signatures, we imaged each pointing separately at $6 \mathrm{~cm}$ and $3 \mathrm{~cm}$. The images of each pointing at both frequencies were largely depolarized, so mosaicing was not the cause of the depolarization seen in Figure 5. To see what effect decreasing the resolution of the images had we made images with 1.5 times and 2.0 times the resolution of the VLA images and an image with the resolution of a 100-m single dish telescope (e.g., the Effelsberg 100-m telescope or the Green Bank Telescope). Decreasing the resolution did not increase the amount of visible polarization, so the resolution of these images was not the cause of the depolarization. To see if bandwidth depolarization is the cause of the depolarization, we imaged each IF separately and compared their polarized emission and determined that there was no significant depolarization associated with combining the two IFs for imaging. Based on these experiments it does not seem likely that instrumental/imaging effects are the cause of the depolarization.

The lack of polarization in Figure 5 could also be due to the properties of the galaxy itself including depolarization effects internal to the galaxy or insufficient sensitivity. Depo-

larization effects internal to the galaxy, e.g., Sokoloff et al. (1998), decrease as ones goes to 
shorter wavelengths. Therefore, the image at $3 \mathrm{~cm}$ should be largely free of depolarization effects while still having appreciable synchrotron emission (see Sections [3.1] and 4.3). The $3 \mathrm{~cm}$ polarization image does not show appreciably more polarization than the $20 \mathrm{~cm}$ polarization image, so internal depolarization effects do not seem to be leading to depolarization at $20 \mathrm{~cm}$ and $6 \mathrm{~cm}$. In Section 4.5.1, we use our polarization data to constrain the uniform magnetic field strength.

\subsection{Comparison with Previous Observations}

Our data represent the first observations of the polarized radio continuum emission from NGC 4214. There are, however, three previous studies of the radio continuum emission of NGC 4214: Allsopp (1979), Beck et al. (2000), and MacKenty et al. (2000).

The earliest interferometric observations of the radio continuum emission from NGC 4214 were done at $20 \mathrm{~cm}$ by Allsopp (1979). Despite their much larger beam (58 by $\left.96^{\prime \prime}\right)$ and higher noise levels, the image derived from these observations has the same overall shape as our higher resolution and better sensitivity image. The emission to the southeast at $12^{\mathrm{h}} 15^{\mathrm{m}} 34^{\mathrm{s}}, 36^{\circ} 20^{\prime}$ that they suggest is a background object is actually clearly part of the main radio continuum distribution in our higher resolution observations and is associated with faint $\mathrm{H} \alpha$ and optical emission.

Beck et al. (2000) observed NGC 4214 with the VLA at $20 \mathrm{~cm}, 6 \mathrm{~cm}, 3.6 \mathrm{~cm}$, and $2 \mathrm{~cm}$ with a resolution of $\sim 2^{\prime \prime}$. By optimizing their observations for higher resolution, however, they resolve out most of the extended structure seen in Figure1, Beck et al. (2000), however, are able to resolve NGC 4214-I and NGC 4214-II into several point sources with hints of more extended emission, particularly in NGC 4214-I. They find that the spectral indices that they derive between $6 \mathrm{~cm}$ and $2 \mathrm{~cm}$ are rising, i.e., positive, and attribute this to the free-free emission becoming optically thick. They estimate that the turnover frequency for NGC 4214 is closer to $15 \mathrm{GHz}$ than to $5 \mathrm{GHz}$. We do not identify any rising spectrum sources between 6 and $3 \mathrm{~cm}$. The beam of our lower resolution observations includes both optically thick sources and extended emission so we would not expect to be able to identify individual optically thick sources.

The $20 \mathrm{~cm}$ and $6 \mathrm{~cm}$ radio continuum observations in MacKenty et al. (2000) are lower resolution $\left(\sim 4^{\prime \prime}\right)$ than those in Beck et al. (2000), but still higher resolution than our observations. Again, the higher resolution of these observations compared to ours allows them to resolve NGC 4214-I and NGC 4214-II into several point sources with hints of extended

emission, but resolves out much of the diffuse emission. MacKenty et al. (2000) were able 
to identify one of the points sources in NGC 4214-I as a supernova remnant based on its spectral index and the properties of the associated optical emission, showing that supernovae have had enough time to produce appreciable synchrotron radiation in NGC 4214.

\section{Discussion}

\subsection{Comparison of the Radio Continuum Emission with Emission at Other Wavelengths}

In Figures 6, 7, and 8, we compare the distribution of the radio continuum emission from NGC 4214 with the distribution of its optical and $\mathrm{H} \alpha$ emission. Figure 6 is a three-color image relating the $\mathrm{H} \alpha$ (red), optical U band (blue), and optical V band (green) to the $20 \mathrm{~cm}$ radio continuum emission. In Figures 7 and 8 , we provide more detailed comparisons of the $20 \mathrm{~cm}, 6 \mathrm{~cm}$, and $3 \mathrm{~cm}$ emission with the $\mathrm{H} \alpha$ and optical $\mathrm{V}$ band, respectively.

From Figure 7, we can see that the radio continuum at $3 \mathrm{~cm}$ closely follows the distribution of the $\mathrm{H} \alpha$ emission. This correspondence shows that the $3 \mathrm{~cm}$ radio continuum emission has a large contribution from thermal emission. As the wavelength of our observations increases, we see more diffuse radio continuum emission. In the northern half of the galaxy, there is diffuse emission that corresponds to the faint $\mathrm{H} \alpha$ filaments at $12^{\mathrm{h}} 15^{\mathrm{m}} 37^{\mathrm{s}}$, $36^{\circ} 20^{\prime} 45^{\prime \prime}$. In the south, there is some diffuse emission that does not closely follow the $\mathrm{H} \alpha$ distribution at $12^{\mathrm{h}} 15^{\mathrm{m}} 45^{\mathrm{s}}, 36^{\circ} 18^{\prime} 00^{\prime \prime}$ and $12^{\mathrm{h}} 15^{\mathrm{m}} 47^{\mathrm{s}}, 36^{\circ} 18^{\prime} 45^{\prime \prime}$; these regions have a large contribution from synchrotron emission. The western tip of the diffuse synchrotron region $\left(12^{\mathrm{h}} 15^{\mathrm{m}} 38^{\mathrm{s}}, 36^{\circ} 22^{\prime} 30^{\prime \prime}\right)$ is associated with $\mathrm{H} \alpha$ emission and some faint 6 and $3 \mathrm{~cm}$ emission. This part of the region is likely dominated by thermal emission. The remainder of the diffuse synchrotron region does not have any corresponding $\mathrm{H} \alpha$ emission or any appreciable emission at 3 or $6 \mathrm{~cm}$, showing that is it dominated by non-thermal emission.

Figure 8 shows that the radio continuum emission is confined to the central regions of this galaxy. We see that the radio continuum emission, the $\mathrm{H} \alpha$ emission, and the stellar emission are all coincident in a bar-shaped region at the center of the galaxy. The diffuse synchrotron region $\left(12^{\mathrm{h}} 15^{\mathrm{m}} 38^{\mathrm{s}}, 36^{\circ} 22^{\prime} 30^{\prime \prime}\right)$ has stars associated with it. This correlation leads us to believe that this feature is an older star formation region. The noticeably resolved, optical object in the center of the region is green in Figure 6, which implies its emission is dominated by optical $\mathrm{V}$ band emission and thus belongs to an older stellar population. The expected lifetime for the free-free emission is about $10 \mathrm{Myr}$ (approximately the lifetime of the most massive stars). The synchrotron emission lifetime is set by the confinement time 
for cosmic rays, i.e., the time it takes for cosmic rays to diffuse out of a galaxy 5 For our Galaxy, this timescale is on the order of 10 Myr. In Section 4.6, we estimate that this time scale is also approximately 10 Myr for NGC 4214 (although see the cavaets discussed in Section 4.6). There is a delay expected between the synchrotron emission and the free-free emission since the first supernovae do not appear until 3.5 Myr after the initial burst of star formation. This argument suggests that there can be synchrotron emission in a region after the free-free emission has dissipated. Note that in this picture, we are assuming that the cosmic rays have not diffused very far from their sources on timescales of 10 Myr.

The timescales for free-free and synchrotron emission allow us to estimate the age of the star formation episode that formed the diffuse synchrotron region. The eastern half of the region has formed stars greater than 10 Myr ago so the free-free emission has dissipated, but less than 13.5 Myr ago, so the cosmic rays are still confined to the galaxy and producing synchrotron emission. The western half has formed stars within $10 \mathrm{Myr}$ and thus still has both synchrotron and free-free emission. The lack of emission in diffuse synchrotron region at 6 and $3 \mathrm{~cm}$ is due to the decrease in synchrotron intensity as the wavelength decreases (i.e., as the frequency increases). Assuming a spectral index of -0.7 and using the intensity of the diffuse synchrotron emission at $20 \mathrm{~cm}$, the synchrotron emission from this patch would be below the $3 \sigma$ detection limit at $6 \mathrm{~cm}$.

\subsection{Fraction of Thermal Emission}

To determine the magnetic field strength, we need to determine how much of the radio continuum emission is due to non-thermal (synchrotron) emission and how much is due to thermal (bremsstrahlung) emission. Since the electrons that produce the thermal emission in the radio are the same electrons that produce $\mathrm{H} \alpha$ in the optical, we use a flux calibrated $\mathrm{H} \alpha$ image kindly provided by D. Hunter (Hunter \& Elmegreen 2004) to estimate the thermal contribution to the radio emission. We use the approximate expression derived by Hunt et al. (2004) to convert the $\mathrm{H} \alpha$ fluxes to radio continuum fluxes

$$
\left(\frac{f_{\nu}}{\mathrm{mJy}}\right)=1.16\left(1+\frac{n\left(H e^{+}\right)}{n\left(H^{+}\right)}\right)\left(\frac{T}{10^{4} \mathrm{~K}}\right)^{0.617}\left(\frac{\nu}{\mathrm{GHz}}\right)^{-0.1}\left(\frac{f_{H \alpha}}{10^{-12} \mathrm{erg} \mathrm{cm}^{-2} \mathrm{~s}^{-1}}\right)
$$

where $f_{\nu}$ is the radio continuum flux, $n\left(\mathrm{He}^{+}\right) / n\left(\mathrm{H}^{+}\right)$is the ratio of ionized helium to ionized hydrogen, $T$ is the temperature of the region, $\nu$ is the frequency of the radio continuum

${ }^{5}$ Small distortions in the galactic magnetic field randomly scatter cosmic rays. See Dogiel \& Breitschwerdt (2009) for a review of the relevant physics. 
observations, and $f_{H \alpha}$ is the $\mathrm{H} \alpha$ flux. These expressions are valid for temperatures between $10,000 \mathrm{~K}$ and $20,000 \mathrm{~K}$ and densities between $100 \mathrm{~cm}^{-3}$ and $1000 \mathrm{~cm}^{-3}$. We use a value of 0.086 for $n\left(H e^{+}\right) / n\left(H^{+}\right)$and a temperature of 10,000 K (Kobulnicky \& Skillman 1996). The synchrotron flux is then

$$
F_{n t h}=F_{t o t}\left(1-\frac{F_{t h}}{F_{t o t}}\right)
$$

where $F_{n t h}$ is the flux of the synchrotron (non-thermal) emission, $F_{\text {tot }}$ is the total flux observed, and $F_{t h}$ is the thermal flux calculated using Equation (11). Any values of $F_{t h} / F_{t o t}$ greater than one or below zero are set to one and zero, respectively. In practice, this affects only a few edge pixels.

We correct the $\mathrm{H} \alpha$ flux for foreground extinction using the value derived from the maps of Schlegel et al. (1998): $A_{R} \sim A_{H \alpha} \sim 0.058$. We do not correct for the internal extinction of NGC 4214, which varies as a function of position in the galaxy (Maiz-Apellaniz et al. 1998). Lisenfeld et al. (2008) find that the internal extinction of NGC 4214 for $\mathrm{H} \alpha$ is between 0.3 and 0.7 magnitudes. The values of high internal extinction in this galaxy are slightly offset from the peaks in the $\mathrm{H} \alpha$ emission. Due to our low resolution $\left(\sim 14^{\prime \prime}\right)$, both the peaks of thermal radio/ $\mathrm{H} \alpha$ emission and the regions of high extinction fall within the same beam. Not correcting for the effect of internal extinction could us to underestimate the flux of the free-free emission by a factor of 1.3 to 1.9 and thus to overestimate the synchrotron flux by factors of 1.25 to 2 for regions that have 0.3 magnitudes of extinction and by factors 2 to 3 for regions that have 0.7 magnitudes of extinction. The lower limits in both cases refer to the flux peaks. Given the value of $\alpha$ used in our calculation of the magnetic field (see Section 4.3 for details), this will change our magnetic field estimate by 9 to 40\%, with the mean change closer to $9 \%$. Correcting the free-free emission also has an effect on the measured polarization of the emission. If the $\mathrm{H} \alpha$ data is not corrected for extinction, the flux of the free-free emission will be an underestimate, so the intensity of the synchrotron will be an overestimate. Then the percent polarization of the synchrotron emission, which is related to the ratio of the uniform field to the random field, will be an underestimate.

Our thermal flux estimates as a function of position in NGC 4214 are shown in Figure 9 .

\subsection{Magnetic Field Strength}

We can calculate the magnetic field strength using the estimated synchrotron flux from Section 4.2, We use the revised equipartition estimate of the magnetic field strength given 
in Beck \& Krause (2005)

$$
B_{t}=\left[\frac{4 \pi(1-2 \alpha)\left(K_{0}+1\right) I_{\nu} E_{p}^{1+2 \alpha}\left(\nu / 2 c_{1}\right)^{-\alpha}}{(-2 \alpha-1) c_{2}(\alpha) l c_{4}}\right]^{1 /(3-\alpha)}
$$

where $B_{t}$ is the total equipartition magnetic field in $\mathrm{G}, \alpha$ is the spectral index of the emission 6 , $K_{0}$ is the number density ratio of the protons to the electrons, $E_{p}$ is the rest energy of the proton, $c_{1}$ is a constant equal to $6.26428 \times 10^{18} \mathrm{erg}^{-2} \mathrm{~s}^{-1} \mathrm{G}^{-1}, c_{2}$ is a constant tabulated on page 232 of Pacholczyk (1970) as $c_{5}, l$ is the line of sight through the galaxy, and $c_{4}$ is a function correcting for the inclination of the region with respect to the sky. Since our polarization observations shown in Section 3.3 do not reveal a large-scale uniform field, we use the version of $c_{4}$ for the case of a completely random, isotropic field

$$
c_{4}=\left(\frac{2}{3}\right)^{(1-\alpha) / 2} .
$$

In this case, $c_{4}$ does not explicitly depend on the inclination, but instead represents an average over all possible orientations for the random field.

We need to carefully consider which frequency has the best estimate of the synchrotron flux. In Section 3.1, we see hints that the radio continuum spectrum of NGC 4214 may be affected by either free-free absorption of synchrotron emission and/or synchrotron losses, although we do not have adequate data at higher and lower frequencies to constrain either model. Free-free absorption of synchrotron emission would cause the $20 \mathrm{~cm}$ synchrotron flux to be underestimated. Cosmic rays also lose energy via synchrotron emission if they are not reaccelerated. This effects the higher energy cosmic rays more than the lower energy cosmic rays and would thus cause the $3 \mathrm{~cm}$ synchrotron flux to be underestimated. Therefore, the $6 \mathrm{~cm}$ image represents the best wavelength for us to determine the magnetic field strength of NGC 4214. We use a typical value for $\alpha$ of -0.7 . Given the uncertainties in the mechanisms responsible for shaping the radio continuum spectrum of NGC 4214, it is better to use a typical value than a value derived from the data. We note that the spectral index between the $20 \mathrm{~cm}$ and $3 \mathrm{~cm}$ synchrotron fluxes for our data is -0.6 , which is very close to the typical value.

We can use the following expression from Ott et al. (2001) to estimate the scale height of NGC 4214's disk from neutral hydrogen observations and obtain the line of sight distance through NGC 4214's disk

$$
h[\mathrm{pc}]=5.79 \times 10^{21}\left(\frac{\sigma_{g a s}}{\mathrm{~km} \mathrm{~s}^{-1}}\right)^{2}\left(\frac{N_{H I}}{\mathrm{~cm}^{-2}}\right)^{-1}\left(\frac{\rho_{H I}}{\rho_{\text {tot }}}\right)
$$

\footnotetext{
${ }^{6}$ We use the opposite sign convention for $\alpha$ as Beck \& Krause (2005).
} 
where $h$ is the scale height, $\sigma_{\text {gas }}$ is the velocity dispersion of the neutral gas, $N_{H I}$ is the column density of the neutral gas, and $\rho_{H I} / \rho_{t o t}$ is the ratio of the density of neutral gas to the total density. From Allsopp (1979), we know that $M_{H I} / M_{t o t}$ is 0.3 , so since $M_{H I} / M_{t o t} \sim$ $\rho_{H I} / \rho_{\text {tot }}=0.3$. Allsopp $(1979)$ also gives us a column density for the neutral gas of $8 \times$ $10^{20} \mathrm{~cm}^{-2}$ and a velocity dispersion of $10 \mathrm{~km} \mathrm{~s}^{-1}$. These values give a scale height for this disk of 200 pc, which agrees with the estimate of the disk scale height by Maíz-Apellániz et al. (1999). Given the uncertain but close to face-on inclination of NGC 4214 we take $i=30^{\circ}$.

The scale height of the magnetic field may be larger than the scale height of the HI. In the Milky Way, the scale height of the HI is $1.6 \mathrm{kpc}$ (Marasco \& Fraternali 2011), while the scale height of the synchrotron emission is $4.6 \mathrm{kpc}$ at the solar circle (Ferrière 2001). Unfortunately, the face-on orientation of NGC 4214 and the lack of information about its overall field topology prevent us from determining the scale height of the synchrotron emission from first principles. We can estimate the effect of a much larger magnetic field scale height on the magnetic field strength estimates for NGC 4214 using the difference between the Milky Way $\mathrm{HI}$ and synchrotron scale height and Equation (3). If the synchrotron scale height of NGC 4214 is three times larger than the HI scale height, then the magnetic field strength estimate above will be 1.3 times bigger than the actual magnetic field strength.

Our estimate of the magnetic field strength in NGC 4214 is given in Figure 10. The magnetic field strength peaks near the center of the galaxy with a value of $30 \mu \mathrm{G}$ and decreases to a value of $10 \mu \mathrm{G}$ at the edge of the galaxy. To get an idea of the uncertainties in these estimates, we have varied $K_{0}$ to $40, \alpha$ by 0.1 , changed the estimated synchrotron emission by a factor of 2 , changed the line of sight distance through the galaxy by $20 \%$, and changed the distance by $20 \%$. With these uncertainities in the initial parameters, we get

an uncertainty for the central magnetic field strength of $9.5 \mu \mathrm{G}$ and an uncertainty for the magnetic field strength on the edges of $3 \mu \mathrm{G}$.

\subsection{Importance of the Magnetic Field in the ISM of NGC 4214}

To determine the relative importance of the magnetic field in the larger context of the interstellar medium of NGC 4214, we calculate total magnetic pressure as well as the magnetic pressure in the inner and outer regions of NGC 4214 and compared this pressure to the thermal pressure from the hot ionized gas, the thermal and turbulent pressure in HII regions, and the gravitational "pressure". These values, along with the values and equations used to calculate them, are given in Table 4.

From these estimates, we can see that the total hot gas pressure, the total magnetic 
pressure, and the gravitational "pressure" are the same order of magnitude, with the hot gas having the largest pressure and the magnetic field the lowest 7 The thermal and turbulent pressures of the HII regions, however, dominate the pressure balance in the central star forming regions (although not the galaxy as a whole). Wilcots \& Thurow (2001) found that the energy in the ionized gas was comparable to that injected via stellar winds. The ISM pressure balance of NGC 4214 is similar to that of NGC 1569 (Kepley et al. 2010); the turbulent and thermal pressures of the HII regions dominate the pressure balance of NGC 1569 in the central star forming regions, while the magnetic field, hot gas, and gravity are approximately equal. Although the magnetic field strength in both galaxies is comparable to that of larger spiral galaxies, neither NGC 4214 nor NGC 1569 appear to be magnetically overpressured compared to their gravitational potential. The magnetic field of NGC 4214 may play a role in shaping any outflow of gas from this galaxy as in NGC 1569 (Kepley et al. 2010), but given the orientation of NGC 4214, we are not able to determine this with our observations (see Section 4.5.3).

\subsection{The Structure of the Magnetic Field of NGC 4214}

\subsubsection{Uniform Field Using Polarization Information}

Using our data, we can calculate an upper limit on the uniform magnetic field strength in NGC 4214. One can approximate the percent polarization as

$$
p=\frac{P}{I}=p_{0} \frac{B_{u, \perp}^{2}}{B_{t, \perp}^{2}}
$$

where $p$ is the percent polarization, $P$ is the intensity of the polarized emission, $I$ is total intensity of the synchrotron emission, $p_{0}$ is the intrinsic polarization of synchrotron emission $(\sim 0.75), B_{u, \perp}$ is the uniform magnetic field in the plane of the sky, and $B_{t, \perp}$ is the total magnetic field in the plane of the sky (Burn 1966; Beck et al. 2003). For NGC 4214, we calculated the total field in Section 4.3 assuming that the field was entirely random, so Equation (6) becomes

$$
p=p_{0} \frac{B_{u, \perp}^{2}}{\frac{2}{3} B_{t}^{2}} .
$$

Assuming a value of $40 \%$ thermal emission at $6 \mathrm{~cm}$ (see Section 4.2), we would detect at the $3 \sigma$ level $30 \%$ polarization at 12 times the noise level (third contour in Figure 5 ). In

\footnotetext{
${ }^{7}$ Since the gravitational pressure was estimated using the Virial Theorem, it should only be compared to the pressures for the entire system, not the pressures for individual regions.
} 
Section 4.3, we estimate the total magnetic field strength at the 3rd contour to be $15 \mu \mathrm{G} 8$ Using Equation (7), we then get an upper limit on the strength of the uniform component of the magnetic field in the plane of the sky of $8 \mu \mathrm{G}$. The large thermal component of the radio continuum emission of NGC 4214 reduces our sensitivity to the uniform field. If the radio continuum emission in NGC 4214 was all synchrotron, our upper limit on the uniform magnetic field strength would be $6 \mu \mathrm{G}$.

From this analysis, we conclude that we do not detect a uniform component of the disk magnetic field of NGC 4214 greater than $8 \mu \mathrm{G}$ on size scales larger than our resolution (200 pc). The disk of NGC 4214 may have a uniform field, but it would have to be weaker than $8 \mu \mathrm{G}$. There could be structure in the magnetic field on size scales smaller than $200 \mathrm{pc}$, but our observations cannot resolve these structures.

\subsubsection{Could the Shape of the Diffuse Synchrotron Region Be Due to a Uniform Field?}

As a star-forming region ages, the relativistic electrons diffuse away from their birthplaces along magnetic field lines. In a completely random field, one would expect that the electrons would diffuse outward isotropically. If there is a uniform component to the field, however, the relativistic electrons will preferentially diffuse along the field lines rather than across them. The diffuse synchrotron region in NGC 4214 is elongated along a position angle of about 60 degrees, which suggests that there is a uniform magnetic field component in that direction.

We can use the distance between the stars and the end of the region (490 pc) and the probable age of the region $(10 \mathrm{Myr})$ to estimate the speed at which the relativistic electrons diffused. This gives a lower limit on the diffusion speed of $4.7 \times 10^{6} \mathrm{~cm} \mathrm{~s}^{-1}$. Assuming that relativistic electrons are moving at the Alfvén speed $\left(v_{A}\right)$

$$
v_{A}^{2}=\frac{B^{2}}{4 \pi \rho}
$$

where $B$ is the magnetic field strength, $\rho$ is the density, and all quantities are in cgs units, if we have an estimate of the density in the region then we can get a lower limit for the strength of the magnetic field responsible for diffusing the cosmic rays. The speed of the cosmic rays is coupled to the density of the thermal electrons, so we can use the $\mathrm{H} \alpha$ image described above to get an estimate of the density of the thermal electrons in the diffuse synchrotron region. In the diffuse synchrotron region there is $4.11 \times 10^{-6} \mathrm{erg}^{-2} \mathrm{~s}^{-1}$ sterad $^{-1}$ of emission. From

\footnotetext{
${ }^{8}$ Recall that this field strength was derived in Section 4.3 assuming that the field was entirely random.
} 
Spitzer (1978),

$$
\operatorname{EM}\left[\mathrm{pc} \mathrm{cm}^{-6}\right]=\frac{\int I_{\nu} \mathrm{d} \nu \lambda}{2.46 \times 10^{17} h c \alpha_{3,2}}
$$

where EM is the emission measure, $I_{\nu}$ is the specific intensity, $\alpha_{3,2}$ is the production coefficient for the $\mathrm{H} \alpha$ line, and all values (unless noted) are in cgs. We use a value of $\alpha_{3,2}$ of $11.7 \times 10^{-14}$ which is valid for 10,000 K. This gives us an emission measure for the region of $47 \mathrm{pc} \mathrm{cm}^{-6}$. If we assume that NGC 4214 is nearly face-on, we use the line of sight distance through the disk derived in Section 4.3 to convert the emission measure to a number density of thermal electrons. This calculation gives a number density for the electrons of $0.34 \mathrm{~cm}^{-3}$. Assuming that the ionized gas has the same number of electrons and protons gives a density for the ionized gas of $5.7 \times 10^{-25} \mathrm{~g} \mathrm{~cm}^{-3}$. The lower limit on the total magnetic field strength can then be derived using

$$
B=v_{A} \sqrt{4 \pi \rho} .
$$

Using our values of $v_{A}$ and $\rho$, the lower limit on the total magnetic field strength in the diffuse synchrotron region is $13 \mu \mathrm{G}$. This field strength is the same as the field strength derived for the diffuse region from the $20 \mathrm{~cm}$ data using the assumptions described in Section 4.3. At $20 \mathrm{~cm}$, we are sensitive to polarizations greater than $26 \%$ in the diffuse synchrotron region. Using the magnetic field strength determined from the synchrotron emission $(13 \mu \mathrm{G})$, we get an upper limit on the uniform field strength of $7.6 \mu \mathrm{G}$.

We can use the ratio of the length of the region to the width of the region to derive the ratio of the uniform magnetic field strength $\left(B_{u}\right)$ to the random magnetic field strength $\left(B_{r}\right)$ and compare it to the limits on $B_{u} / B_{r}$ from our data. Let us take $y$ to be the direction along the diffuse synchrotron region and $x$ to be the direction across it, the equation of the magnetic field to be $\mathbf{B}=\hat{y} B_{y}+\hat{x} B_{x}, X$ to be half the total width of the region, and $Y$ to be half the total length of the region. $B_{y}$ and $B_{x}$ are both constant in a correlation length $l$, but $B_{x}$ can be positive or negative. In this picture, $B_{y}$ corresponds to the uniform component and $B_{x}$ corresponds to the random component. Then the equation of a field line is

$$
\frac{\mathrm{dy}}{\mathrm{dx}}=\frac{B_{y}}{B_{x}} .
$$

The displacement in $y$ when the field line has gone a distance $l$ in $x$ is

$$
\Delta y=\frac{l B_{y}}{B_{x}} .
$$

The cosmic rays stream at the Alfvén speed, so the time to travel the distance $l$ is $\tau=l / v_{A x}$, where $v_{A x}=B_{x} /(4 \pi \rho)^{1 / 2}$. After a time $t$, the cosmic rays have taken $N=t / \tau$ random steps of length $l$ in the $x$ direction, so the $r m s$ displacement of the cosmic rays, $X$, is 
$l N^{1 / 2}=\left(l v_{A x} t\right)^{1 / 2}$. The displacement in the $y$ direction is just $N \Delta y=N l B_{y} / B_{x}$. Then the elongation of the cloud is

$$
\frac{Y}{X}=\left(\frac{v_{A x} t}{l}\right)^{1 / 2} \frac{B_{y}}{B_{x}}=N^{1 / 2} \frac{B_{y}}{B_{x}} .
$$

We can estimate $N$ from the strength of the random field derived above from our data $(12.9 \mu \mathrm{G})$, the density of the region $\left(0.34 \mathrm{~cm}^{-3}\right)$, the age of the region (10 Myr), and the outer length scale for turbulence (90 pc in the LMC; Gaensler et al. 2005). This gives a value for $N$ of approximately 6 . The measured values of $\mathrm{X}$ and $\mathrm{Y}$ are $235 \mathrm{pc}$ and $491 \mathrm{pc}$, respectively, which gives a value for $Y / X$ of 2.1. With our estimate of $N$, the ratio $B_{y} / B_{x}=B_{u} / B_{r}$ is 0.85. From our data, the ratio of $B_{u} / B_{r}$ allowed by our data is 0.69 , which is close enough to the ratio estimated from the size of the region to make our scenario that the diffuse synchrotron region is elongated by a uniform field plausible.

\subsubsection{Possible Structure in the Halo of NGC 4214}

The analysis in Sections 4.5.1 and 4.5.2 only applies to the structure of the magnetic field in the disk of NGC 4214. The nearly face-on orientation of NGC 4214 does not allow us to determine whether NGC 4214 has a uniform magnetic field oriented along a galactic outflow like that seen in NGC 1569 (Kepley et al. 2010). From estimates of the mechanical energy in the wind $\left(L_{\text {mech }} \sim 10^{40}-10^{41} \mathrm{erg} \mathrm{s}^{-1}\right)$, it appears that it is possible for NGC 4214 to experience a blow-out (Ott et al. 2005b). Therefore, NGC 4214 might have magnetic field structure perpendicular to its disk in its halo. Detection of this halo field may be possible using the dense grid of background rotation measures produced by the Square Kilometer Array (Beck \& Gaensler 2004).

We would like to note that while in principle the rotation measure of the background source to the northeast of the main body of emission could be used to estimate the magnetic field direction and strength along the line of sight, i.e., through the disk, unfortunately there is no $\mathrm{H} \alpha$ emission in the region of the galaxy associated with the background source, so we would be unable to determine a field strength. In addition, there are no other nearby background sources to allow us to estimate an average rotation measure for background sources in that region of the sky. 


\subsection{Cosmic Ray Lifetimes}

Using the arguments from Section 4.5.2, we can also estimate a timescale for cosmic ray confinement in NGC 4214. Assuming that a supernova goes off at the midplane of the disk, the cosmic rays would have to travel 200 pc to to escape the disk. Since cosmic rays diffuse at the Alfvén velocity, the total displacement of the cosmic rays, $L$, in a time $t$ is

$$
L=\left(l v_{A} t\right)^{1 / 2}=\frac{(l B t)^{1 / 2}}{(4 \pi \rho)^{1 / 4}} .
$$

The timescale for the cosmic rays to escape the disk is then

$$
t=\frac{X^{2}\left(4 \pi n_{e} m_{H}\right)^{1 / 2}}{l B}
$$

where $n_{e}$ is the number density of electrons and $m_{H}$ is the mass of a hydrogen atom. We estimate $n_{e}$ to be $1 \mathrm{~cm}^{-3}, B$ to be $20 \mu \mathrm{G}$, and take $l$ to be the outer length scale of the turbulence, which Gaensler et al. (2005) found to be $90 \mathrm{pc}$ in the LMC. Using these parameters, the timescale for the diffusion of cosmic rays out of the disk of NGC 4214 is approximately $10 \mathrm{Myr}$ - comparable to the diffusion time in the Milky Way. If the scale height of the synchrotron emission is much larger, as discussed at the end of Section 4.3, this timescale could be as long as $67 \mathrm{Myr}$. NGC 4214 may have a wind in its central portion Ott et al. (2005b) which would shorten the cosmic ray confinement time (Everett et al. 2008, 2010). Detailed modeling of the effect of a central wind on the cosmic ray confinement time in NGC 4214 is beyond the scope of the paper.

Synchrotron losses will further limit the lifetime of synchrotron emission. To see if the synchrotron loss timescale will dominate over the cosmic ray confinement time, we calculate the lifetime of cosmic ray electrons due to synchrotron losses. The power $P$ in $\operatorname{erg~s}^{-1}$ radiated by synchrotron emission is

$$
P=\frac{4}{3} \sigma_{T} c \beta^{2} \gamma^{2} \frac{B^{2}}{8 \pi}
$$

where $\sigma_{T}$ is the Thomson cross-section, $c$ is the speed of light, $\beta$ is $v / c$, and $\gamma$ is $\left(1-\beta^{2}\right)^{-1 / 2}$. Since cosmic rays are traveling very close to the speed of light, we can assume $\beta \approx 1$. Assuming that all the emission is produced at the critical frequency

$$
\nu_{c}=\frac{3}{4 \pi} \gamma^{2} \frac{q B}{m c} \sin \alpha
$$

and using the relation $E=\gamma m c^{2}$, we find that the energy of the electron at the critical frequency is

$$
E=\left(\frac{4 \pi m_{e}^{3} c^{5} \nu_{c}}{3 q B}\right)^{1 / 2}
$$


where the factor of $\sin \alpha$ has been ignored since it is a factor on the order of one. Dividing the result of Equation (18) by Equation (16) gives an estimate for the synchrotron loss timescale. For emission at $20 \mathrm{~cm}(1.4 \mathrm{GHz})$ and a magnetic field strength of $10 \mu \mathrm{G}$ (the field strength in the diffuse synchrotron region), the synchrotron loss timescale for a cosmic ray electron is $44 \mathrm{Myr}$. At $6 \mathrm{~cm}(8.5 \mathrm{GHz})$, the lifetime of the cosmic rays due to synchrotron losses is shorter - $18 \mathrm{Myr}$. Therefore, at $20 \mathrm{~cm}$ in the diffuse synchrotron region, the confinement time is shorter than the synchrotron loss timescale and thus the confinement time dominates. (Although if the synchrotron scale height is 600 pc rather than 200 pc, the synchrotron loss time will dominate.) At lower frequencies and larger magnetic fields, the synchrotron loss time scale decreases so that in the center of the galaxy with a magnetic field of $30 \mu \mathrm{G}$ and at $3 \mathrm{~cm}$, the synchrotron loss timescale may be as short at $6 \mathrm{Myr}$.

\subsection{Generating the Magnetic Field}

To evaluate the possibility of an $\alpha-\omega$ dynamo operating in NGC 4214, we compare the shear and turbulence in NGC 4214 to that of a larger galaxy, where an $\alpha-\omega$ dynamo may be operating: the Milky Way.

First, we evaluate the effect of differential rotation by calculating the shear in both systems. Shear is defined as $\mathrm{d} \Omega / \mathrm{d} r$, which for a flat rotation curve is just $v / r^{2}$. At the solar radius $(8 \mathrm{kpc})$, the rotation speed is $220 \mathrm{~km} \mathrm{~s}^{-1}$, so the shear in the Milky Way is about $3.4 \mathrm{~km} \mathrm{~s}^{-1} \mathrm{kpc}^{-2}$. In Figure 11, we show the absolute value of the shear in NGC 4214 as a function of radius. To calculate the shear, we used the neutral hydrogen rotation curve for NGC 4214 given in Allsopp (1979), corrected for the distance and inclination used in this paper. The shear in NGC 4214 varies significantly below a radius of $2 \mathrm{kpc}$, but the average value at these radii is close to the Milky Way value. At a radius of $2 \mathrm{kpc}$, the shear begins to increase, reaching a maximum of $12 \mathrm{~km} \mathrm{~s}^{-1} \mathrm{kpc}^{-2}$ at a radius of about $3 \mathrm{kpc}$. The shear then begins to decrease and by $5 \mathrm{kpc}$ is at or below the shear estimated at the solar radius in the Milky Way. We would note that the diffuse synchrotron region discussed in Section 4.5 .2 is located in the region of maximum shear about $2.5 \mathrm{kpc}$ from the center of NGC 4214. According to Rayleigh's Criterion, the quantity $\Omega r^{2}$ must increase as a function of $r$ for a rotation curve to be stable. This is satisfied for the $\Omega$ shown in Figure 11 everywhere except in $3 \mathrm{kpc}<r<5.5 \mathrm{kpc}$, but the observational uncertainties are large enough that the departure from stability, which is slight, is most likely due to error.

To evaluate the turbulent component of the dynamo process, we assume that the turbulence in these galaxies is generated by supernovae and use the supernova rate as a proxy for turbulence. In the Milky Way, the supernova rate from type II supernovae is $27 \mathrm{Myr}^{-1} \mathrm{kpc}^{-2}$ 
(Ferrière 2001). For NGC 4214, we can determine the supernova rate from the star formation rate. Hunter \& Elmegreen (2004) determine a star formation rate per unit area for NGC 4214 of $5.0 \times 10^{3} \mathrm{M}_{\odot} \mathrm{yr}^{-1} \mathrm{kpc}^{-2}$. Assuming a Salpeter IMF, that the range of masses for stars is $0.8 \mathrm{M}_{\odot}$ to $150 \mathrm{M}_{\odot}$, and that only stars above $8 \mathrm{M}_{\odot}$ become Type II supernovae, we obtain a rate for the formation of stars greater than $8 \mathrm{M}_{\odot}$ of $85 \mathrm{Myr}^{-1} \mathrm{kpc}^{-2}$. Assuming a massive star lifetime of $30 \mathrm{Myr}$ and that the burst of star formation in NGC 4214 began 4 Myr ago (MacKenty et al. 2000), that gives a supernova rate of $11.3 \mathrm{Myr}^{-1} \mathrm{kpc}^{-2}$.

Comparing the shear and the supernova rates between the Milky Way and NGC 4214, we see that NGC 4214 has a similar level of shear, but a lower supernova rate by a factor of 2. This rough calculation may indicate that additional turbulence needs to be injected by supernovae in order to provide the turbulence necessary for the shear to generate a largescale field. Given that the episode of star formation in NGC 4214 started relatively recently and that it seems to be continuously producing stars, we anticipate that supernovae will be able to generate additional turbulence as the galaxy ages. In shear-dominated turbulence, one expects that the field would be oriented (although not necessarily directed) azimuthally. The region of diffuse synchrotron emission is located near the shear maximum in NGC 4214, which suggests that the magnetic field in this region is oriented by the shear, but not necessarily directed as it would be in a large scale dynamo.

Amplification by an $\alpha-\omega$ mechanism is rather slow, so various authors (e.g. Parker 1992) have proposed mechanisms to decrease the time needed to amplify the field. If a largescale field could be generated for NGC 4214 using a faster dynamo like the one proposed for NGC 4449 (Otmianowska-Mazur et al. 2000), it may have not had time to develop yet or it may have been disrupted by recent star formation. Note that we cannot probe magnetic field structure on size scales less than $200 \mathrm{pc}$ due to the resolution of our observations.

\section{Conclusions}

In this paper, we have presented the deepest radio continuum polarization images of the irregular galaxy NGC 4214 to date. Below we summarize the overall conclusions of our work.

The global radio continuum spectrum for NGC 4214 is convex, which suggests that either free-free absorption of synchrotron emission and/or free-free emission or synchrotron losses are occurring. We fit the global radio continuum spectrum of NGC 4214 with a single power law with a spectral index of -0.43 because of the offsets between our data and the archival data, the limited wavelength coverage of our data, and the heterogenity of the archival data. 
This spectral index indicates that the radio continuum emission in NGC 4214 is a mix of thermal and non-thermal emission. The spectral indices of the radio continuum peaks are flat, with the younger region (NGC 4214-II) having the flatter spectral index. Between the radio continuum peaks, the spectral indices are steeper, showing that synchrotron emission plays a more important role there. We do not detect any significant polarized emission in NGC 4214 at size scales greater than 200 pc and conclude that this is due to the intrinsic nature of the galaxy rather than instrumental/imaging effects or internal depolarization.

We find that the radio continuum emission at $3 \mathrm{~cm}$ is correlated with the $\mathrm{H} \alpha$ emission from this galaxy, while the radio continuum emission at $20 \mathrm{~cm}$ is more extended. The central regions of NGC 4214 are approximately $50 \%$ thermal at $3 \mathrm{~cm}$. North of the main body of radio continuum emission, we detect a region of diffuse synchrotron radiation associated with a group of stars in NGC 4214 and speculate that this region is a relic of previous star formation. Based the relative timescales of free-free and synchrotron emission, we estimate an age for this region of between 10 and 13.5 Myr.

We estimate the fraction of thermal radio continuum emission in NGC 4214 using H $\alpha$ images. Using this estimate, we calculate the estimated intensity of the synchrotron radiation to estimate the magnetic field strength of NGC 4214. The field strength reaches a maximum of $30 \pm 9.5 \mu \mathrm{G}$ in the center of the galaxy and tapers off to $10 \pm 3 \mu \mathrm{G}$ at the edges. Comparing the pressures of the various components of the ISM of NGC 4214, we find that the hot gas pressure, the magnetic pressure, and the gravitational "pressure" all the same order of magnitude. In the central star forming regions of NGC 4214, the thermal and turbulent pressure of the HII regions dominate the pressure balance of this galaxy. The pressure balance in NGC 4214 is similar to that of another irregular galaxy: NGC 1569. In both galaxies, star formation plays the dominant role in shaping the ISM. Although the magnetic field strength in both galaxies is comparable to that of larger spiral galaxies, neither NGC 4214 nor NGC 1569 appear to be magnetically overpressured compared to their gravitational potential.

Using our polarization data, we place an upper limit on the uniform magnetic field strength of $8 \mu \mathrm{G}$ on size scales greater than $200 \mathrm{pc}$. We speculate that the elongation of the diffuse synchrotron emission is due to a uniform field component. Using simple estimates of the Alfvén speed and the density of the region along with upper limits on the polarization from our data, we place an upper limit of $7.6 \mu \mathrm{G}$ on the uniform field strength in this region and calculate a total field strength of $13 \mu \mathrm{G}$ and a random field strength of $11 \mu \mathrm{G}$. These limits are consistent with the observed elongation of the diffuse synchrotron region.

We use the simple model developed in Section 4.5.2 to estimate the cosmic ray confinement time in NGC 4214 and find it to be appropriately $10 \mathrm{Myr}$, comparable to the diffusion 
time in the Milky Way. Synchrotron losses and a galactic wind may further limit the lifetimes of cosmic rays in the galaxy.

Finally, we investigate whether an $\alpha-\omega$ dynamo could operate in NGC 4214 by comparing its shear and supernova rate to that of the Milky Way. We find that the shear in NGC 4214 is comparable to the Milky Way, but that the supernova rate is half that of the Milky Way. We suggest that there is not enough turbulence yet in the NGC 4214 to drive an $\alpha-\omega$ dynamo, but that as the star formation in NGC 4214 progresses the additional turbulence will allow an $\alpha-\omega$ dynamo to operate.

Of the low mass irregular galaxies with measured magnetic fields, we find that the magnetic field of NGC 4214 most resembles that of IC 10, i.e., strong with some evidence of uniform fields. In a future paper, we will investigate the properties of all observed magnetic fields in irregular galaxies and attempt to create an integrated picture of how they are generated and sustained in these galaxies.

The authors thank the referee for their careful report. They also gratefully acknowledge Deidre Hunter for providing the optical and $\mathrm{H} \alpha$ images used in this paper. AAK would like to thank Jay Gallagher, Snežana Stanimirović, Dan McCammon, Bryan Gaensler, Sui Ann Mao, Jake Simon, and Phil Arras for helpful discussions. AAK acknowledges support from a National Science Foundation Graduate Fellowship, GBT student support awards (GSSP070001,-0002,-0003,-0019), and a Wisconsin Space Grant Consortium Graduate Fellowship. EMW acknowledges support from a grant from the National Science Foundation (AST0708002).

This research has made use of the NASA/IPAC Extragalactic Database (NED) which is operated by the Jet Propulsion Laboratory, California Institute of Technology, under contract with the National Aeronautics and Space Administration, and of NASA's Astrophysics Data System Bibliographic Services.

Facilities: VLA

\section{A. Deriving the Gravitational Pressure}

To derive the gravitational pressure, we start with the Virial Theorem

$$
2 K+U=0
$$

where $K$ is the kinetic energy and $U$ is the potential energy. Here we assume that the density distribution of NGC 4214 can be approximated by an isothermal sphere with density 
distribution

$$
\rho(r)=\rho_{0}\left(\frac{r_{0}}{r}\right)^{2}
$$

(Kepley et al. 2007; van Evmeren et al. 2009), where $\rho_{0}$ and $r_{0}$ are the central density and core radius, $\rho$ is density, and $r$ is radius. Note that the rotation curves of galaxies are more typically fit with a pseudo-isothermal sphere

$$
\rho(r)=\rho_{0}\left[1+\left(\frac{r_{0}}{r}\right)^{2}\right]^{-1}
$$

which does not have infinite density at zero radius like Equation (A2) does. As $r$ becomes large, the density from Equation (A2) tends to the density from Equation (A3). We are measuring the potential far from the center of the galaxy, so we assume that Equation (A2) applies, which yields a much more analytically tractable result. The mass interior to radius $r, M(r)$, is then just

$$
\begin{aligned}
M(r) & =\int_{0}^{r} 4 \pi \rho\left(r^{\prime}\right)\left(r^{\prime}\right)^{2} \mathrm{~d} r^{\prime} \\
& =4 \pi \rho_{0} r_{0}^{2} r
\end{aligned}
$$

The potential energy of an individual shell, $\mathrm{dU}$, is then

$$
\begin{aligned}
\mathrm{dU} & =\frac{-G M(r) \mathrm{d} m}{r} \\
& =\frac{-G M(r) 4 \pi r^{2} \rho(r) \mathrm{d} r}{r} \\
& =-16 \pi^{2} G \rho_{0}^{2} r_{0}^{4} r
\end{aligned}
$$

where $G$ is the gravitational constant. Integrating this expression out to a radius $R$ and substituting for $\rho_{0}$ gives the potential energy, $U$, for an isothermal sphere

$$
U=\frac{-G M^{2}}{R}
$$

For an ideal gas, the kinetic energy is just

$$
K=\frac{3}{2} P V
$$

where $P$ is the pressure and $V$ is the volume of the galaxy. Inserting the expressions for the potential and kinetic energies into the Virial Theorem and dividing by the Boltzmann constant $(k)$ to put the pressure in units of $\mathrm{K} \mathrm{cm}^{-3}$, we obtain the expression

$$
\frac{P}{k}=\frac{G M^{2}}{3 R V k} \text {. }
$$


Since $V=(4 / 3) \pi R^{3}$ and the circular velocity $v$ at radius $R$ in the galaxy is just $v^{2}=G M / R$, Equation (A11) can be rewritten as

$$
\frac{P}{k}=\frac{v^{4}}{4 \pi G R^{2} k}
$$

We would note that regardless of the potential equation we use (isothermal sphere, constant density, $\rho \propto r^{2}$, etc.), the multiplicative factor in front of the potential changes by only a factor of 2 . Since the gravitational pressure is proportional to the total potential energy, changes in the assumed form of the potential only change the gravitational pressure by a factor of a few.

\section{REFERENCES}

Allsopp, N. J. 1979, MNRAS, 188, 765

Beck, R. 2005, in Lecture Notes in Physics, Berlin Springer Verlag, Vol. 664, Cosmic Magnetic Fields, ed. R. Wielebinski \& R. Beck, 41-+

Beck, R., \& Gaensler, B. M. 2004, New Astronomy Review, 48, 1289

Beck, R., Klein, U., \& Wielebinski, R. 1987, A\&A, 186, 95

Beck, R., \& Krause, M. 2005, Astronomische Nachrichten, 326, 414

Beck, R., Shukurov, A., Sokoloff, D., \& Wielebinski, R. 2003, A\&A, 411, 99

Beck, S. C., Turner, J. L., \& Kovo, O. 2000, AJ, 120, 244

Becker, R. H., White, R. L., \& Edwards, A. L. 1991, ApJS, 75, 1

Becker, R. H., White, R. L., \& Helfand, D. J. 1995, ApJ, 450, 559

Burn, B. J. 1966, MNRAS, 133, 67

Chi, X., \& Wolfendale, A. W. 1993, Nature, 362, 610

Chyzy, K. T., Beck, R., Kohle, S., Klein, U., \& Urbanik, M. 2000, A\&A, 356, 757

Chyży, K. T., Beck, R., Kohle, S., Klein, U., \& Urbanik, M. 2000, A\&A, 355, 128

Chyży, K. T., Knapik, J., Bomans, D. J., Klein, U., Beck, R., Soida, M., \& Urbanik, M. 2003, A\&A, 405, 513 
Chyży, K. T., Weżgowiec, M., Beck, R., \& Bomans, D. J. 2011, A\&A, 529, A94+

Condon, J. J., Cotton, W. D., \& Broderick, J. J. 2002, AJ, 124, 675

Cormier, D., Madden, S. C., Hony, S., Contursi, A., Poglitsch, A., Galliano, F., Sturm, E., Doublier, V., Feuchtgruber, H., Galametz, M., Geis, N., de Jong, J., Okumura, K., Panuzzo, P., \& Sauvage, M. 2010, A\&A, 518, L57+

Cox, D. P. 2005, ARA\&A, 43, 337

Dalcanton, J. J., Williams, B. F., Seth, A. C., Dolphin, A., Holtzman, J., Rosema, K., Skillman, E. D., Cole, A., Girardi, L., Gogarten, S. M., Karachentsev, I. D., Olsen, K., Weisz, D., Christensen, C., Freeman, K., Gilbert, K., Gallart, C., Harris, J., Hodge, P., de Jong, R. S., Karachentseva, V., Mateo, M., Stetson, P. B., Tavarez, M., Zaritsky, D., Governato, F., \& Quinn, T. 2009, ApJS, 183, 67

de Avillez, M. A., \& Breitschwerdt, D. 2005, A\&A, 436, 585

de Vaucouleurs, G., de Vaucouleurs, A., Corwin, H. G., Buta, R. J., Paturel, G., \& Fouque, P. 1995, VizieR Online Data Catalog, 7155, 0

Deeg, H.-J., Brinks, E., Duric, N., Klein, U., \& Skillman, E. 1993, ApJ, 410, 626

Dogiel, V., \& Breitschwerdt, D. 2009, ArXiv e-prints

Dopita, M. A., Calzetti, D., Maíz Apellániz, J., Blair, W. P., Long, K. S., Mutchler, M., Whitmore, B. C., Bond, H. E., MacKenty, J., Balick, B., Carollo, M., Disney, M., Frogel, J. A., O’Connell, R., Hall, D., Holtzman, J. A., Kimble, R. A., McCarthy, P., Paresce, F., Saha, A., Walker, A. R., Silk, J., Sirianni, M., Trauger, J., Windhorst, R., \& Young, E. 2010, Ap\&SS, 148

Dressel, L. L., \& Condon, J. J. 1978, ApJS, 36, 53

Drozdovsky, I. O., Schulte-Ladbeck, R. E., Hopp, U., Greggio, L., \& Crone, M. M. 2002, AJ, 124,811

Everett, J. E., Schiller, Q. G., \& Zweibel, E. G. 2010, ApJ, 711, 13

Everett, J. E., Zweibel, E. G., Benjamin, R. A., McCammon, D., Rocks, L., \& Gallagher, III, J. S. 2008, ApJ, 674, 258

Fanelli, M. N., Waller, W. W., Smith, D. A., Freedman, W. L., Madore, B., Neff, S. G., O’Connell, R. W., Roberts, M. S., Bohlin, R., Smith, A. M., \& Stecher, T. P. 1997, ApJ, 481, 735 
Ferrara, A., \& Tolstoy, E. 2000, MNRAS, 313, 291

Ferrière, K. M. 2001, Reviews of Modern Physics, 73, 1031

Gaensler, B. M., Haverkorn, M., Staveley-Smith, L., Dickey, J. M., McClure-Griffiths, N. M., Dickel, J. R., \& Wolleben, M. 2005, Science, 307, 1610

Gregory, P. C., \& Condon, J. J. 1991, ApJS, 75, 1011

Greisen, E., ed. 2008, AIPS Cookbook (NRAO)

Hanasz, M., Kowal, G., Otmianowska-Mazur, K., \& Lesch, H. 2004, ApJ, 605, L33

Hartwell, J. M., Stevens, I. R., Strickland, D. K., Heckman, T. M., \& Summers, L. K. 2004, MNRAS, 348, 406

Haynes, R. F., Klein, U., Wayte, S. R., Wielebinski, R., Murray, J. D., Bajaja, E., Meinert, D., Buczilowski, U. R., Harnett, J. I., Hunt, A. J., Wark, R., \& Sciacca, L. 1991, A\&A, 252, 475

Haynes, R. F., Murray, J. D., Klein, U., \& Wielebinski, R. 1986, A\&A, 159, 22

Hopp, U., Schulte-Ladbeck, R. E., Greggio, L., \& Crone, M. M. 1999, in Astronomical Society of the Pacific Conference Series, Vol. 192, Spectrophotometric Dating of Stars and Galaxies, ed. I. Hubeny, S. Heap, \& R. Cornett, 85-+

Hunt, L. K., Dyer, K. K., Thuan, T. X., \& Ulvestad, J. S. 2004, ApJ, 606, 853

Hunter, D. A., Brinks, E., Elmegreen, B., Rupen, M., Simpson, C., Walter, F., Westpfahl, D., \& Young, L. 2007, in American Astronomical Society Meeting Abstracts, Vol. 211, American Astronomical Society Meeting Abstracts, \#95.06

Hunter, D. A., \& Elmegreen, B. G. 2004, AJ, 128, 2170

-. 2006, ApJS, 162, 49

Hunter, D. A., Elmegreen, B. G., \& Ludka, B. C. 2010, AJ, 139, 447

Hunter, D. A., Elmegreen, B. G., \& Martin, E. 2006, AJ, 132, 801

Kepley, A. A., Mühle, S., Everett, J., Zweibel, E. G., Wilcots, E. M., \& Klein, U. 2010, ApJ, 712,536

Kepley, A. A., Wilcots, E. M., Hunter, D. A., \& Nordgren, T. 2007, AJ, 133, 2242 
Klein, U., Haynes, R. F., Wielebinski, R., \& Meinert, D. 1993, A\&A, 271, 402

Klein, U., Wielebinski, R., \& Thuan, T. X. 1984, A\&A, 141, 241

Kobulnicky, H. A., \& Skillman, E. D. 1996, ApJ, 471, 211

Kulsrud, R. M. 1999, ARA\&A, 37, 37

Kulsrud, R. M., \& Zweibel, E. G. 2008, Reports on Progress in Physics, 71, 046901

Lisenfeld, U., Relano, M., Vilchez, J., Battaner, E., \& Hermelo, I. 2008, ArXiv e-prints, 808

MacKenty, J. W., Maíz-Apellániz, J., Pickens, C. E., Norman, C. A., \& Walborn, N. R. 2000, AJ, 120, 3007

Maíz-Apellániz, J., Cieza, L., \& MacKenty, J. W. 2002, AJ, 123, 1307

Maiz-Apellaniz, J., Mas-Hesse, J. M., Munoz-Tunon, C., Vilchez, J. M., \& Castaneda, H. O. 1998, A\&A, 329, 409

Maíz-Apellániz, J., Muñoz-Tuñón, C., Tenorio-Tagle, G., \& Mas-Hesse, J. M. 1999, A\&A, 343,64

Mao, S. A., Gaensler, B. M., Stanimirović, S., Haverkorn, M., McClure-Griffiths, N. M., Staveley-Smith, L., \& Dickey, J. M. 2008, ApJ, accepted

Marasco, A., \& Fraternali, F. 2011, A\&A, 525, A134+

Martin, C. L. 1998, ApJ, 506, 222

McIntyre, V. J. 1997, Publications of the Astronomical Society of Australia, 14, 122

McMahon, R. G., White, R. L., Helfand, D. J., \& Becker, R. H. 2002, ApJS, 143, 1

Mineshige, S., Shibata, K., \& Shapiro, P. R. 1993, ApJ, 409, 663

Otmianowska-Mazur, K., Chyży, K. T., Soida, M., \& von Linden, S. 2000, A\&A, 359, 29

Ott, J., Walter, F., \& Brinks, E. 2005a, MNRAS, 358, 1423

-. 2005b, MNRAS, 358, 1453

Ott, J., Walter, F., Brinks, E., Van Dyk, S. D., Dirsch, B., \& Klein, U. 2001, AJ, 122, 3070 
Pacholczyk, A. G. 1970, Radio astrophysics. Nonthermal processes in galactic and extragalactic sources (Series of Books in Astronomy and Astrophysics, San Francisco: Freeman, 1970)

Parker, E. N. 1992, ApJ, 401, 137

Sault, R. J., Teuben, P. J., \& Wright, M. C. H. 1995, in Astronomical Society of the Pacific Conference Series, Vol. 77, Astronomical Data Analysis Software and Systems IV, ed. R. A. Shaw, H. E. Payne, \& J. J. E. Hayes, 433-+

Schlegel, D. J., Finkbeiner, D. P., \& Davis, M. 1998, ApJ, 500, 525

Schmitt, H. R., Calzetti, D., Armus, L., Giavalisco, M., Heckman, T. M., Kennicutt, Jr., R. C., Leitherer, C., \& Meurer, G. R. 2006, ApJS, 164, 52

Schwartz, C. M., \& Martin, C. L. 2004, ApJ, 610, 201

Simmons, J. F. L., \& Stewart, B. G. 1985, A\&A, 142, 100

Sokoloff, D. D., Bykov, A. A., Shukurov, A., Berkhuijsen, E. M., Beck, R., \& Poezd, A. D. 1998, MNRAS, 299, 189

Spitzer, L. 1978, Physical processes in the interstellar medium, ed. Spitzer, L.

Springel, V., White, S. D. M., Jenkins, A., Frenk, C. S., Yoshida, N., Gao, L., Navarro, J., Thacker, R., Croton, D., Helly, J., Peacock, J. A., Cole, S., Thomas, P., Couchman, H., Evrard, A., Colberg, J., \& Pearce, F. 2005, Nature, 435, 629

Steidel, C. C., Giavalisco, M., Pettini, M., Dickinson, M., \& Adelberger, K. L. 1996, ApJ, 462, L17+

Subramanian, K. 1998, MNRAS, 294, 718

Tomisaka, K. 1990, ApJ, 361, L5

Tremonti, C. A., Heckman, T. M., Kauffmann, G., Brinchmann, J., Charlot, S., White, S. D. M., Seibert, M., Peng, E. W., Schlegel, D. J., Uomoto, A., Fukugita, M., \& Brinkmann, J. 2004, ApJ, 613, 898

Úbeda, L., Maíz-Apellániz, J., \& MacKenty, J. W. 2007a, AJ, 133, 917

—. 2007b, AJ, 133, 932

Vaillancourt, J. E. 2006, PASP, 118, 1340 
van Eymeren, J., Trachternach, C., Koribalski, B. S., \& Dettmar, R. 2009, A\&A, 505, 1

Wielebinski, R. 2005, in Lecture Notes in Physics, Berlin Springer Verlag, Vol. 664, Cosmic Magnetic Fields, ed. R. Wielebinski \& R. Beck, 89-+

Wilcots, E. M., \& Thurow, J. C. 2001, ApJ, 555, 758

Zweibel, E. G., \& Heiles, C. 1997, Nature, 385, 131 
Table 1. Summary of VLA Observations

\begin{tabular}{llll}
\hline \hline \multicolumn{1}{c}{ Observing Band } & \multicolumn{2}{c}{$20 \mathrm{~cm}$} & \multicolumn{2}{c}{$6 \mathrm{~cm}$} & \multicolumn{2}{c}{$3 \mathrm{~cm}$} \\
\hline Array & $\mathrm{C}$ & $\mathrm{D}$ & $\mathrm{D}$ \\
Date & 2005 Aug 7 & 2005 Dec 5 \& 19 & 2005 Dec 23 \& 24 \\
IF 1 Frequency (GHz) & 1.3649 & 4.8851 & 8.4351 \\
IF 2 Frequency (GHz) & 1.4351 & 4.8351 & 8.4851 \\
Bandwidth/IF (MHz) & 50 & 50 & 50 \\
Number of Channels/IF & 1 & 1 & 1 \\
Primary Calibrator & $1331+305$ & $1331+305$ & $1331+305$ \\
Secondary Calibrator & $1227+365$ & $1146+399$ & $1146+399$ \\
Number of Pointings & 1 & 4 & 16 \\
Field of View (') & 30 & 9 & 5.4 \\
Resolution (") & 12.5 & 14.0 & 8.4 \\
Largest Angular Scale (') & 15 & 5 & 3 \\
Integration Time per Pointing (hours) & 10.0 & 3.73 & 1.2 \\
\hline
\end{tabular}

Table 2. Final Images

\begin{tabular}{cccccc}
\hline \hline $\begin{array}{c}\text { Frequency } \\
\mathrm{GHz}\end{array}$ & $\begin{array}{c}\text { Beam } \\
\prime\end{array}$ & $\begin{array}{c}\text { PA } \\
\circ\end{array}$ & $\begin{array}{c}\sigma_{I} \\
\mu \mathrm{Jy} \mathrm{beam}^{-1}\end{array}$ & $\begin{array}{c}\sigma_{Q} \\
\mu \mathrm{Jy} \mathrm{beam}^{-1}\end{array}$ & $\begin{array}{c}\sigma_{U} \\
\mu \mathrm{Jy} \mathrm{beam}^{-1}\end{array}$ \\
\hline 1.397 & $14.18^{\prime \prime} \times 11.46^{\prime \prime}$ & $-77.3^{\circ}$ & 48.8 & 13.0 & 12.9 \\
4.860 & $14.18^{\prime \prime} \times 11.46^{\prime \prime}$ & $-77.3^{\circ}$ & 25.1 & 17.8 & 18.5 \\
8.460 & $14.18^{\prime \prime} \times 11.46^{\prime \prime}$ & $-77.3^{\circ}$ & 24.1 & 20.7 & 21.0 \\
\hline
\end{tabular}


Table 3. Radio Continuum Flux Spectrum

\begin{tabular}{ccccc}
\hline \hline $\begin{array}{c}\text { Frequency } \\
\text { GHz }\end{array}$ & $\begin{array}{c}\text { Flux } \\
\text { mJy }\end{array}$ & $\begin{array}{c}\text { Flux Error } \\
\text { mJy }\end{array}$ & Telescope & Source \\
\hline 1.40 & 51.5 & 10.3 & VLA & 6 \\
1.40 & 38.3 & 7.7 & VLA/NVSS & 5 \\
2.38 & 36.0 & 3.0 & Arecibo & 4 \\
4.86 & 34.0 & 6.8 & VLA & 6 \\
4.85 & 30.0 & 4.5 & GB 300ft & 2 \\
4.85 & 30.0 & 7.0 & GB 300ft & 3 \\
8.46 & 20.5 & 0.5 & VLA & 1 \\
8.46 & 24.2 & 4.8 & VLA & 6 \\
\hline
\end{tabular}

References. - (1) Schmitt et al. 2006; (2) Becker et al. 1991; (3) Gregory \& Condon 1991; (4) Dressel \& Condon 1978; (5) Condon et al. 2002; (6) This paper

Table 4. Pressures of Various Components of the ISM of NGC 4214

\begin{tabular}{|c|c|c|c|c|}
\hline Component & Input Values & Equation & Reference & $\begin{array}{c}\text { Pressure } \\
10^{5} \mathrm{~K} \mathrm{~cm}^{-3}\end{array}$ \\
\hline Magnetic field (center) & $B=30 \mu \mathrm{G}$ & $B^{2} /(8 \pi k)$ & 1 & 2.6 \\
\hline Magnetic field (edges) & $B=10 \mu \mathrm{G}$ & $B^{2} /(8 \pi k)$ & 1 & 0.3 \\
\hline Magnetic field (total) & $B=13 \mu \mathrm{G}$ & $B^{2} /(8 \pi k)$ & 1 & 0.5 \\
\hline Hot gas (central) & $n_{e}=0.147 \mathrm{~cm}^{-3}, T=2.80 \times 10^{6} \mathrm{~K}$ & $2 n_{e} T$ & 2 & 8.2 \\
\hline Hot gas (edges) & $n_{e}=0.033 \mathrm{~cm}^{-3}, T=2.68 \times 10^{6} \mathrm{~K}$ & $2 n_{e} T$ & 2 & 1.7 \\
\hline Hot gas (total) & $n_{e}=0.064 \mathrm{~cm}^{-3}, T=2.17 \times 10^{6} \mathrm{~K}$ & $2 n_{e} T$ & 2 & 2.8 \\
\hline HII regions (thermal) & $n_{e}=100 \mathrm{~cm}^{-3}, T=10,500 \mathrm{~K}$ & $2 n_{e} T$ & 3 & 10.5 \\
\hline HII regions (turbulent) & $n_{e}=100 \mathrm{~cm}^{-3}, v_{\text {turb }}=50 \mathrm{~km} \mathrm{~s}^{-1}$ & $n_{e} m_{H} v_{\text {turb }}^{2} /(k)$ & 4 & 300 \\
\hline Gravity $^{\mathrm{a}}$ & $v_{\text {rad }}=20 \mathrm{~km} \mathrm{~s}^{-1}, \mathrm{i}=30^{\circ}, \mathrm{r}=1.11 \mathrm{kpc}$ & $\left(v_{r a d} / \sin i\right)^{4} /\left(4 \pi r^{2} G k\right)$ & 5 & 1.9 \\
\hline
\end{tabular}

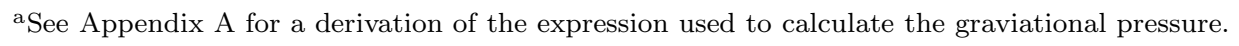

References. - (1) This work; (2) Ott et al. (2005b); (3) Kobulnicky \& Skillman (1996); (4) Wilcots \& Thurow (2001); (5) Allsopp (1979). 


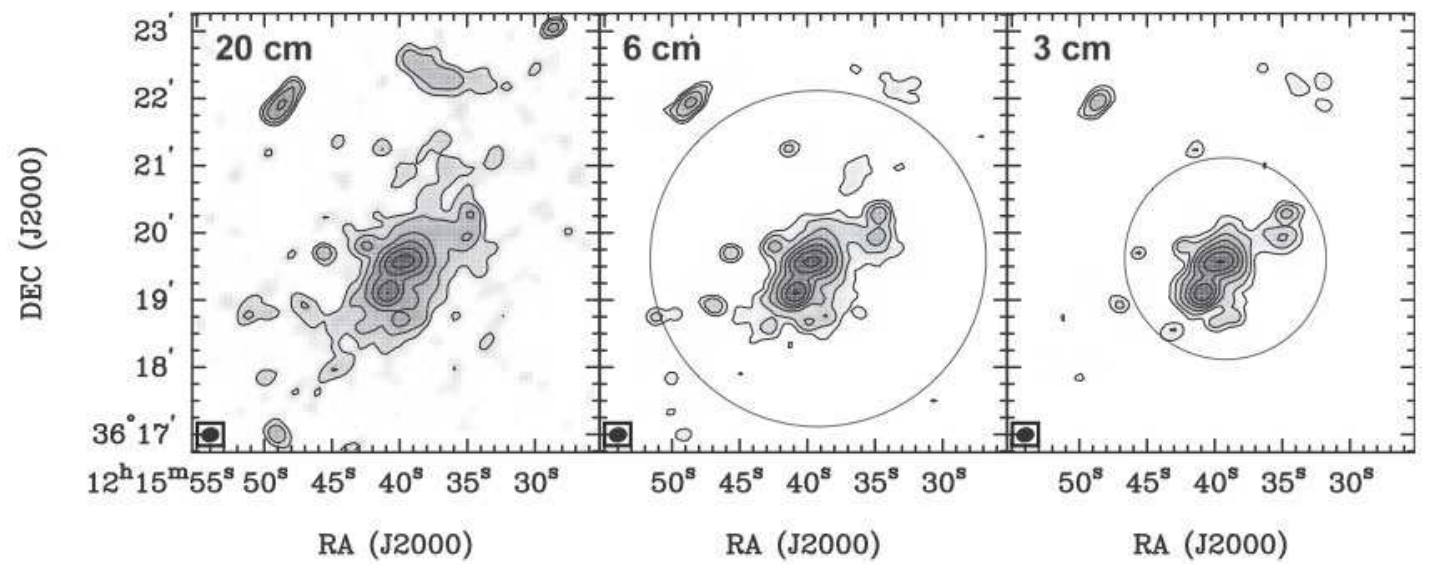

Fig. 1. - Maps of the total intensity radio continuum emission of NGC 4214 at $20 \mathrm{~cm}$ (left panel), $6 \mathrm{~cm}$ (middle panel), and $3 \mathrm{~cm}$ (right panel). The images are all on the same logarithmic scale stretching from $72.3 \mu \mathrm{Jy}_{\mathrm{beam}}{ }^{-1}$ to $20 \mathrm{mJy}$ beam $^{-1}$. The contours are 3 , $6,12,24,48,96,192$, and 384 times the $1 \sigma$ noise level given in Table 2 . The beam is boxed in the lower left corner of each panel and is identical for all three images. The diameter of the circles in the middle and right panels show the largest angular scale imaged by the VLA at $6 \mathrm{~cm}$ and $3 \mathrm{~cm}$, respectively. The largest angular scale imaged by the VLA at $20 \mathrm{~cm}$ is larger than the size of the region shown. 


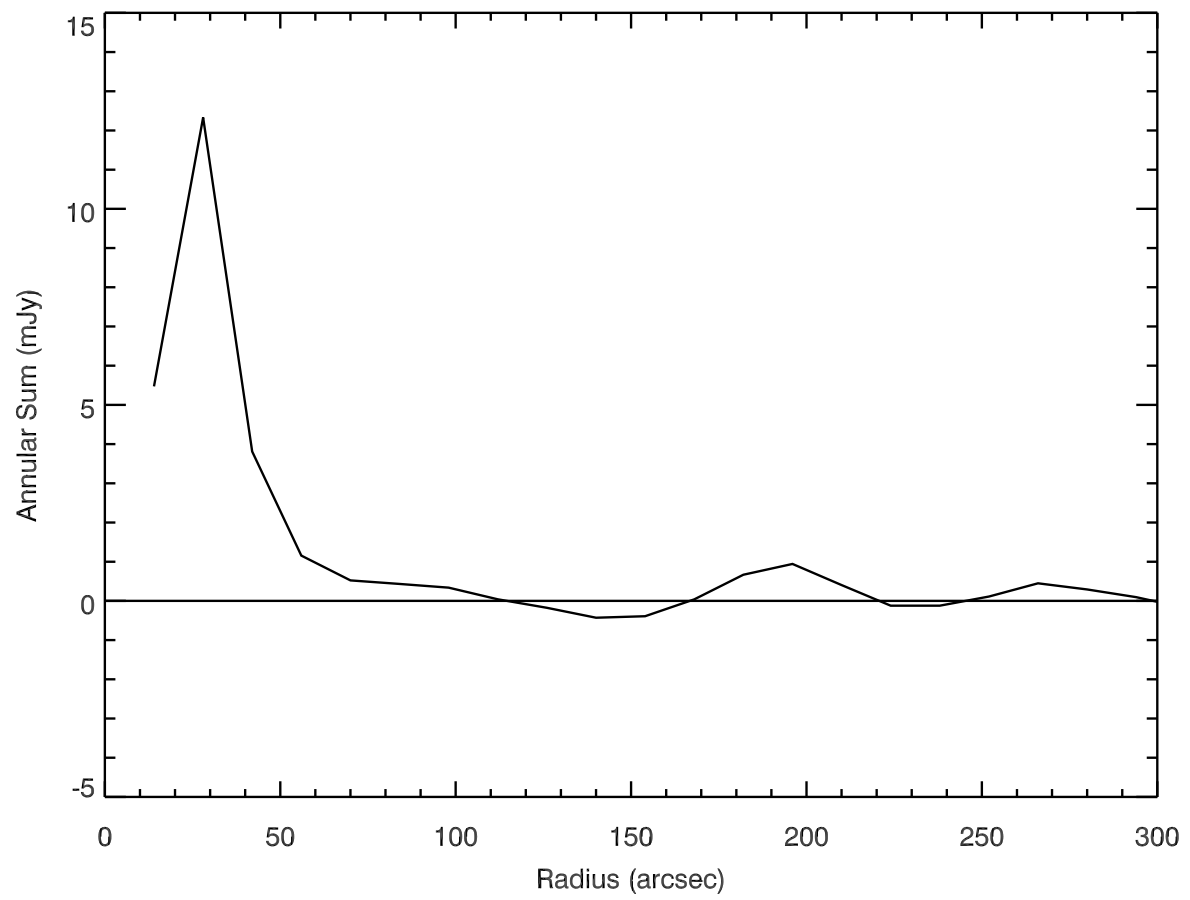

Fig. 2. $-3 \mathrm{~cm}$ radio continuum emission per $14^{\prime \prime}$ annulus about the center of the galaxy as a function of radius. The presence of a large negative bowl of emission, which is not seen here, would indicate that significant flux is resolved out. 


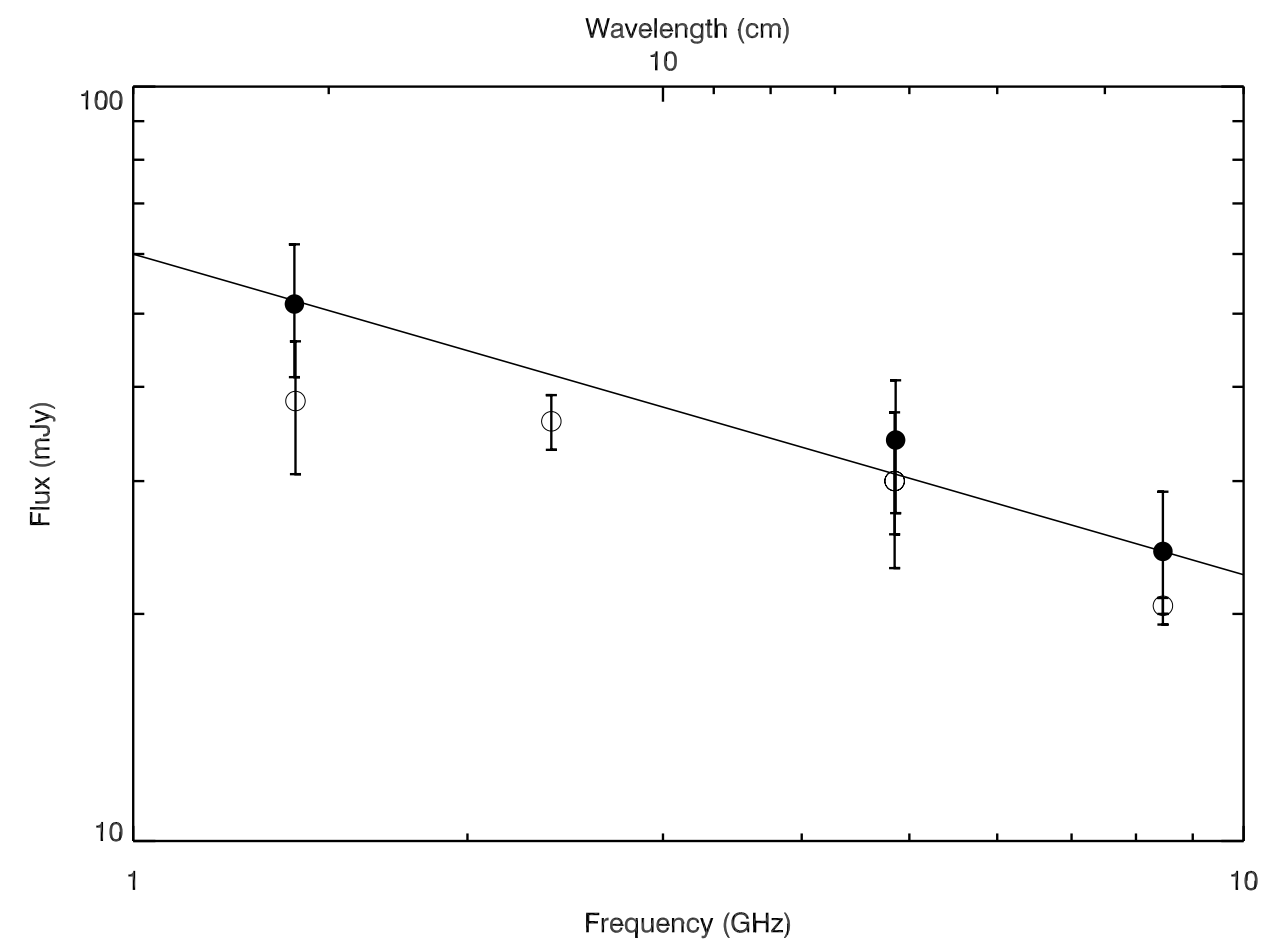

Fig. 3.- Radio continuum spectrum of NGC 4214. The open points are previous measurements and the solid points are our measurements from this paper. The line is the best power law fit to our measurements; see the discussion in $\S 3.1$. 


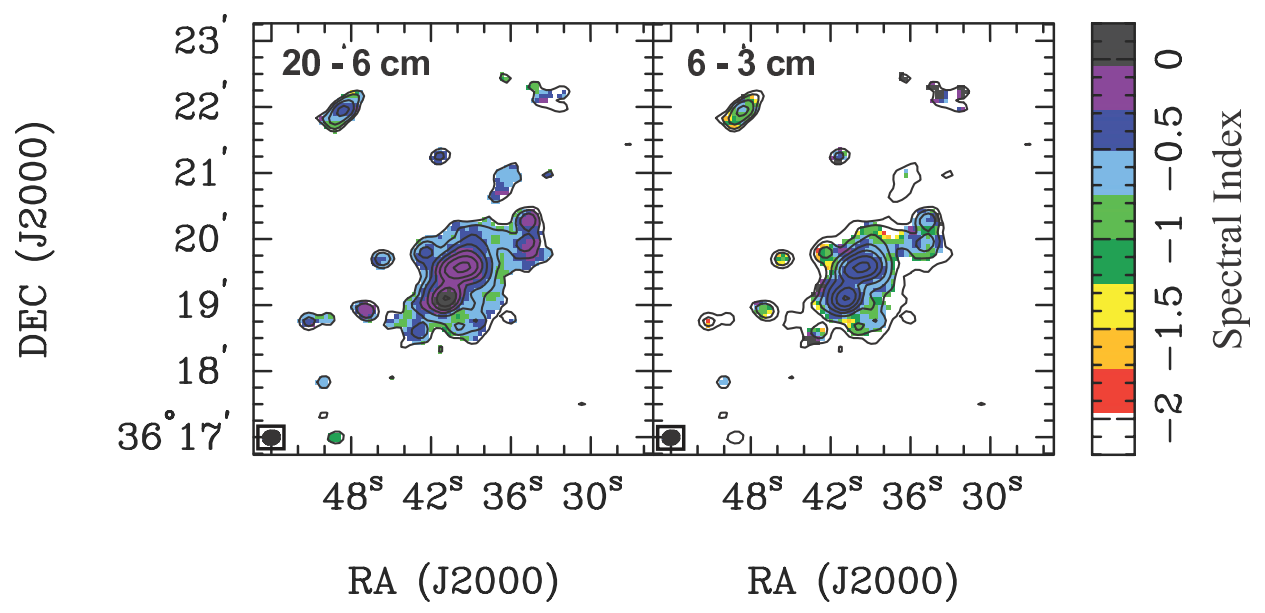

Fig. 4.- Spectral index between the $20 \mathrm{~cm}$ and $6 \mathrm{~cm}$ data (left panel) and between the $6 \mathrm{~cm}$ and $3 \mathrm{~cm}$ data (right panel). Pixels below $3 \sigma$ in the input images were masked before calculation of the spectral indices. The contours show the total intensity radio continuum emission at $6 \mathrm{~cm}$. The contours are 3, 6, 12, 24, 48, 96, 192, and 384 times the $1 \sigma$ noise level given in Table 2. The beam is boxed in the lower left corner of each panel.

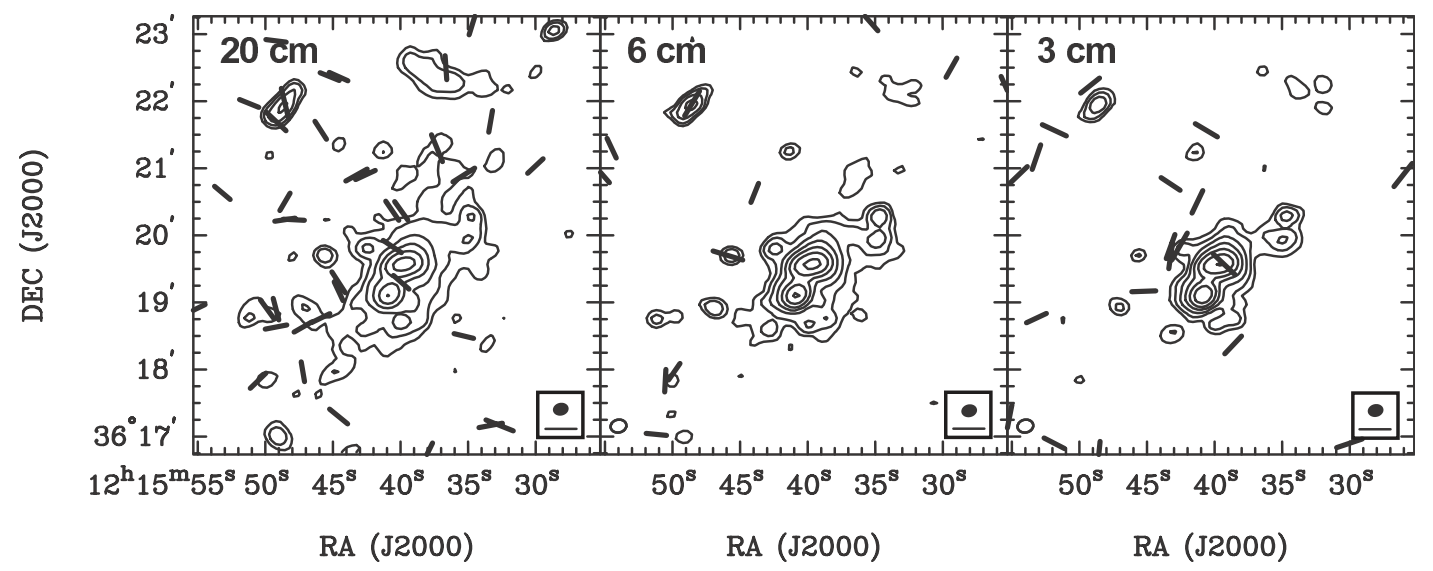

Fig. 5.- Polarized intensity vectors (E-vectors) overlaid on contours showing the total intensity radio continuum emission of NGC 4214 at $20 \mathrm{~cm}$ (left panel), $6 \mathrm{~cm}$ (middle panel), and $3 \mathrm{~cm}$ (right panel). The polarized intensities have been bias corrected and only vectors with a signal to noise greater than 3 are shown. The contours are 3, 6, 12, 24, 48, 96, 192, and 384 times the $1 \sigma$ noise level given in Table 2, The beam and the polarization vector scale bar are boxed in the right hand corner of each panel. The beam is identical for all three images. The length of the polarization vector scale bar corresponds to a polarized intensity of $5.5 \times 10^{-5} \mathrm{Jy}_{\text {beam }}{ }^{-1}$. 


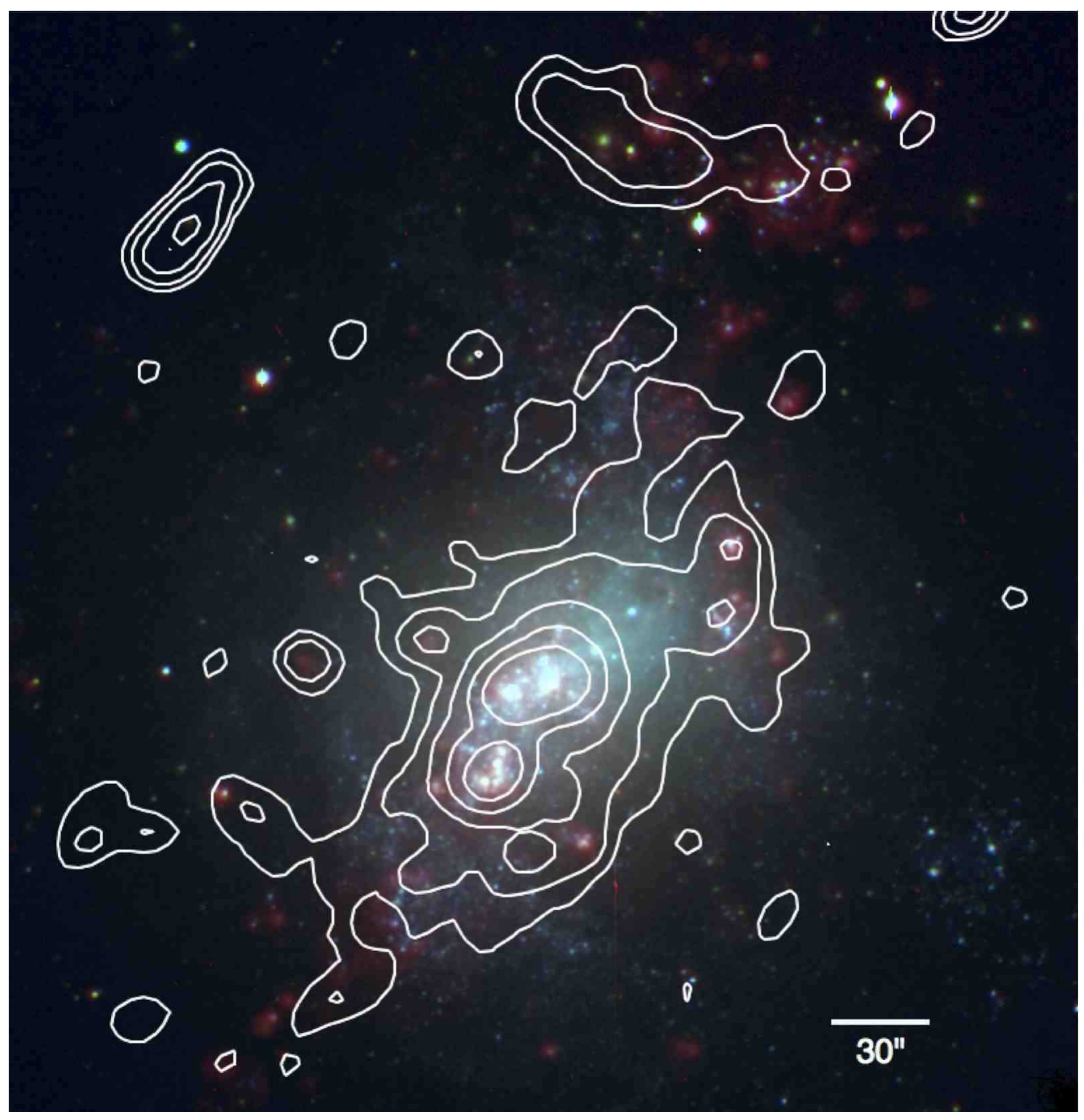

Fig. 6. - $20 \mathrm{~cm}$ total intensity radio continuum emission contours overlaid on a three color optical image of NGC 4214. The optical images are courtesy D. Hunter and red represents $\mathrm{H} \alpha$, blue the $\mathrm{U}$ band optical, and green $\mathrm{V}$ band optical. The contours are 3, 6, 12, 24, 48, 96, 192, and 384 times the $1 \sigma$ noise level given in Table 2 . 


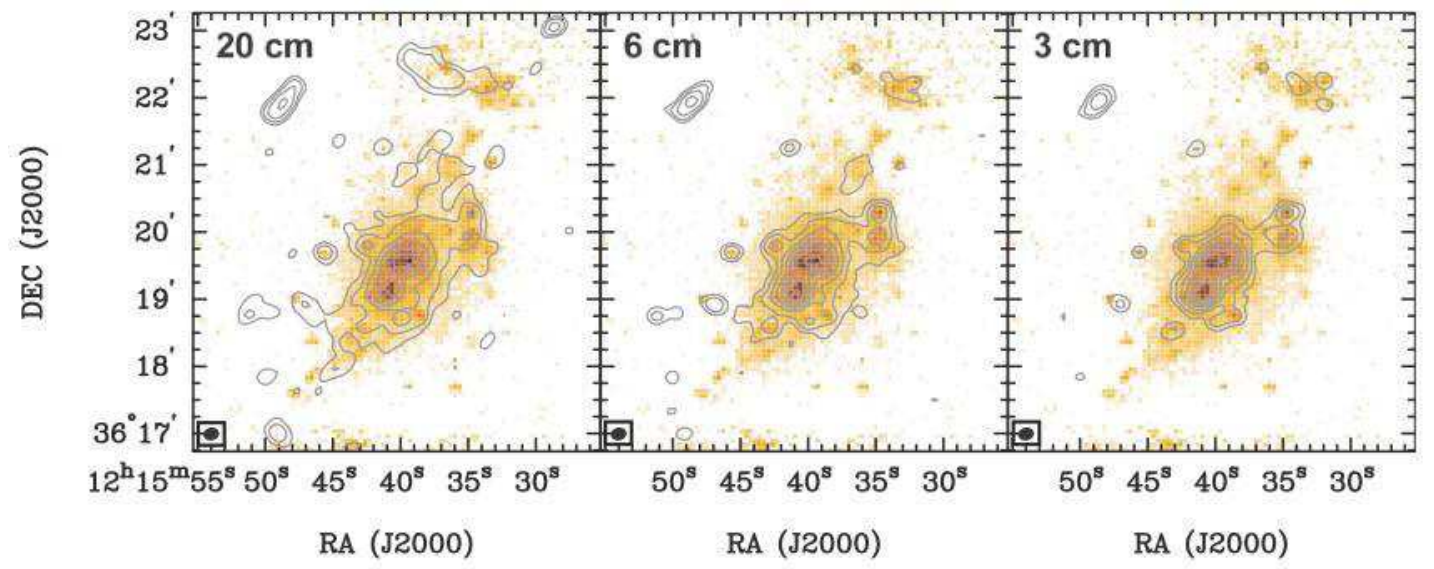

Fig. 7.- Contours of total intensity radio continuum emission at $20 \mathrm{~cm}$ (left panel), $6 \mathrm{~cm}$ (middle panel), and $3 \mathrm{~cm}$ (right panel) overlaid on an $\mathrm{H} \alpha$ image of NGC 4214 kindly provided by Deidre Hunter (Hunter \& Elmegreen 2004). The contours are 3, 6, 12, 24, 48, 96, 192, and 384 times the $1 \sigma$ noise level given in Table 2. The beam is boxed in the lower left corner of each panel.

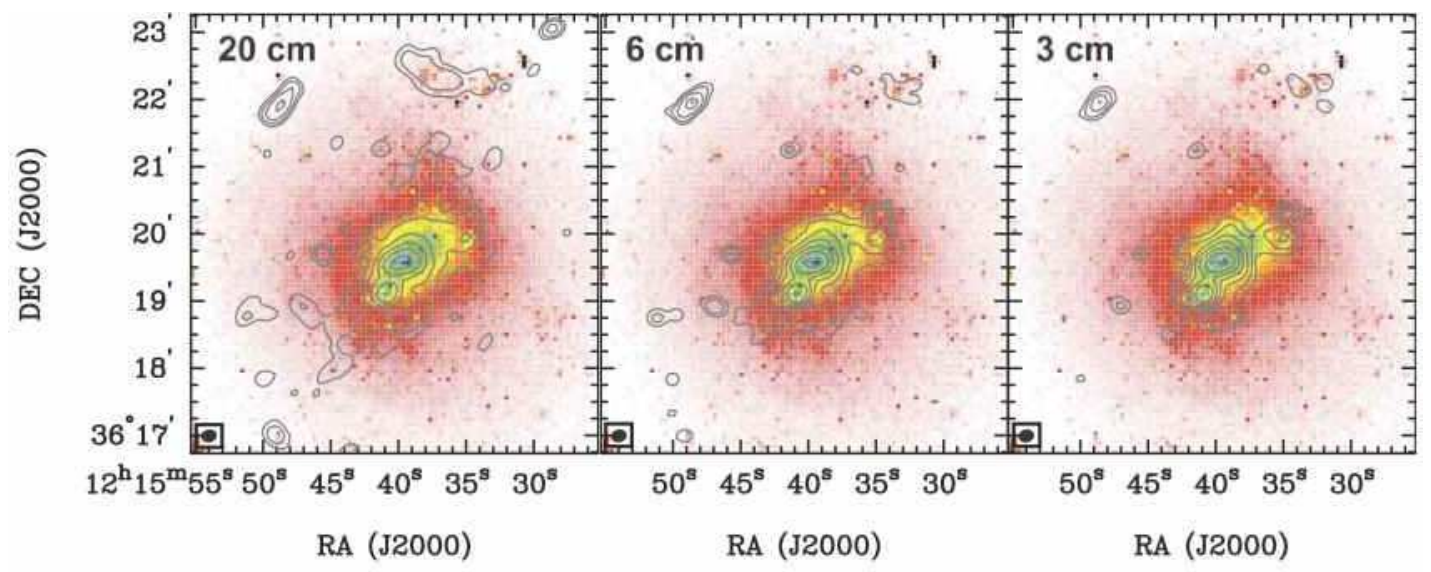

Fig. 8.- Contours of total intensity radio continuum emission at $20 \mathrm{~cm}$ (left panel), $6 \mathrm{~cm}$ (middle panel), and $3 \mathrm{~cm}$ (right panel) overlaid on an optical V band image of NGC 4214 kindly provided by Deidre Hunter (Hunter \& Elmegreen 2006). The contours are 3, 6, 12, $24,48,96,192$, and 384 times the $1 \sigma$ noise level given in Table 2. The beam is boxed in the lower left corner of each panel. 


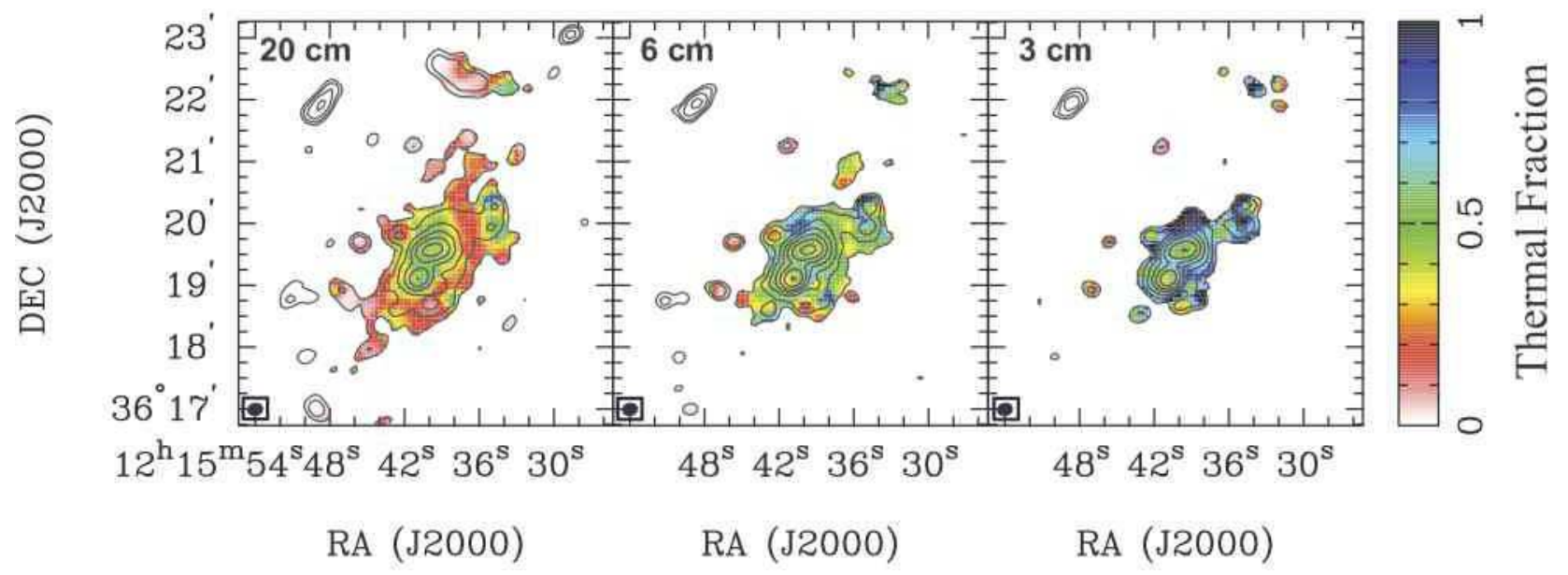

Fig. 9. - Estimated thermal fraction of NGC 4214 with contours of total intensity radio continuum emission at $20 \mathrm{~cm}$ (left panel), $6 \mathrm{~cm}$ (middle panel), and $3 \mathrm{~cm}$ (right panel). The contours are $3,6,12,24,48,96,192$, and 384 times the $1 \sigma$ noise level given in Table 2. The beam is boxed in the lower left corner of each panel. 


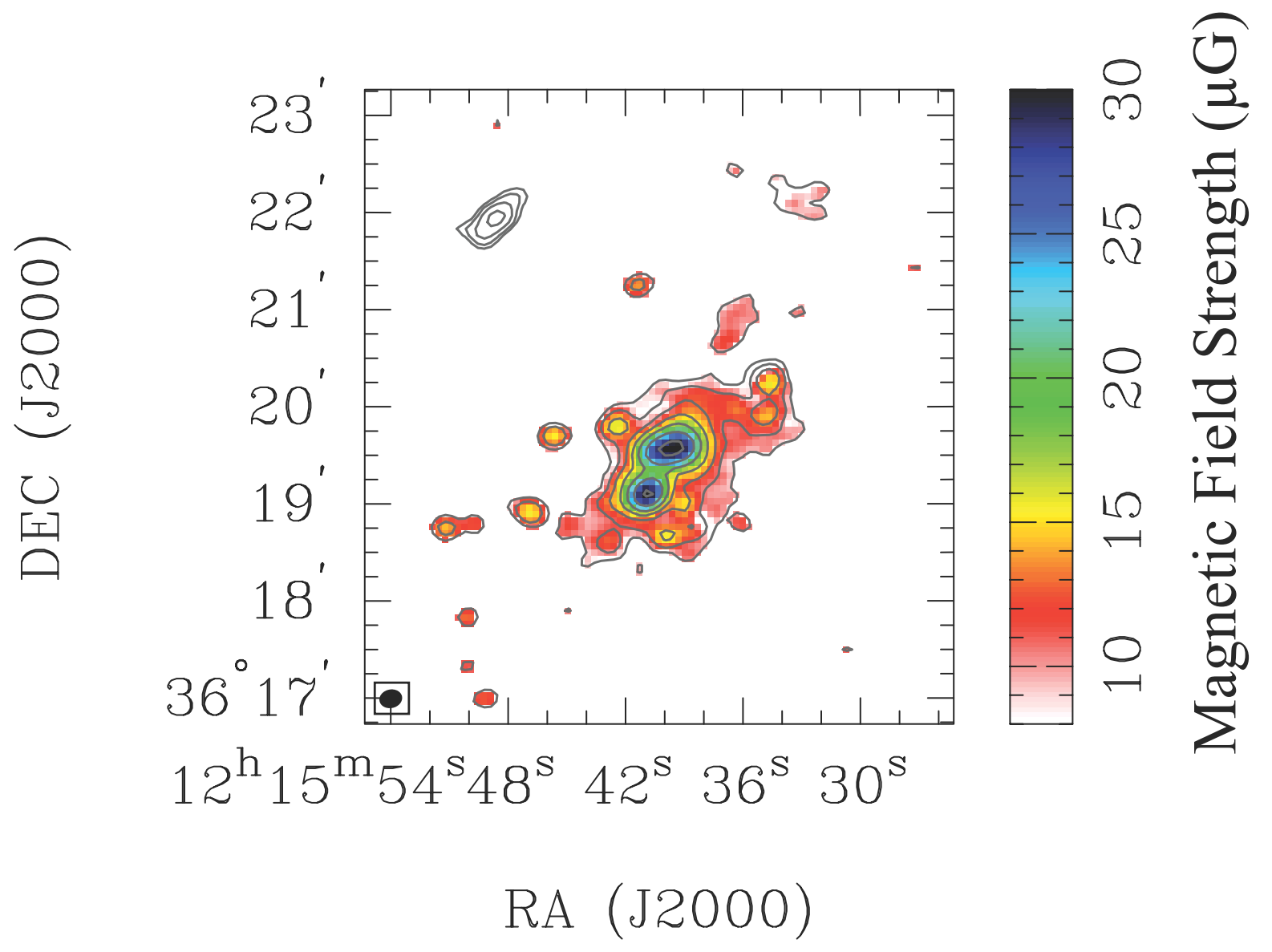

Fig. 10.- Magnetic field strength (in $\mu \mathrm{G}$ ) calculated using the estimated synchrotron emission at $6 \mathrm{~cm}$. The magnetic field has not been calculated for the background AGN at $\left(12^{\mathrm{h}} 15^{\mathrm{m}} 48.9^{\mathrm{s}}, 36^{\circ} 21^{\prime} 54.10^{\prime \prime}\right)$ since it does not belong to NGC 4214 . The beam is boxed in the lower left. 


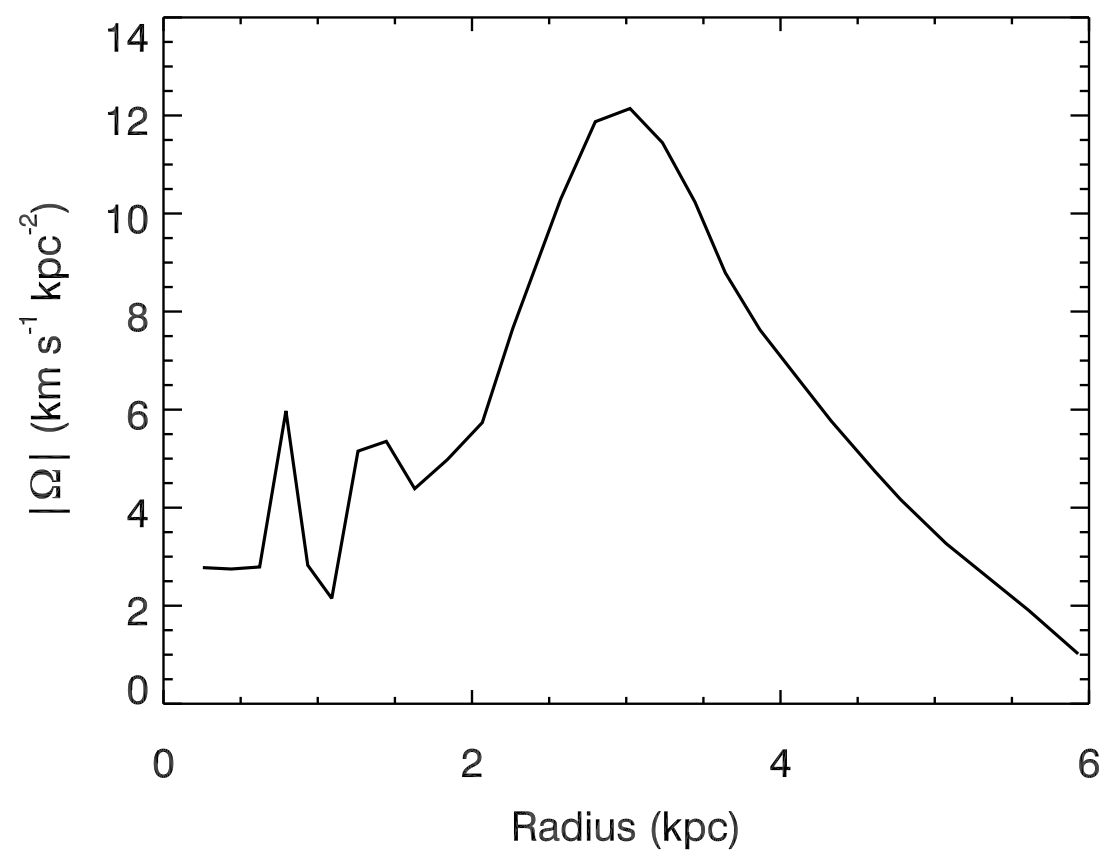

Fig. 11. - Absolute value of the shear calculated using the neutral hydrogen rotation curve from Allsopp (1979), corrected for the distance adopted in this paper and for the inclination of NGC 4214, as a function of radius. 